\title{
Energy Efficient Chip-to-Chip Wireless Interconnection for Heterogeneous Architectures
}

\author{
SRI HARSHA GADE, Indraprastha Institute of Information Technology Delhi, India \\ M. MERAJ AHMED, Rochester Institute of Technology, USA \\ SUJAY DEB, Indraprastha Institute of Information Technology Delhi, India \\ AMLAN GANGULY, Rochester Institute of Technology, USA
}

\begin{abstract}
Heterogeneous multichip architectures have gained significant interest in high-performance computing clusters to cater to a wide range of applications. In particular, heterogeneous systems with multiple multicore CPUs, GPUs, and memory have become common to meet application requirements. The shared resources like interconnection network in such systems pose significant challenges due to the diverse traffic requirements of CPUs and GPUs. Especially, the performance and energy consumption of inter-chip communication have remained a major bottleneck due to limitations imposed by off-chip wired links. To overcome these challenges, we propose a wireless interconnection network to provide energy-efficient, high-performance communication in heterogeneous multi-chip systems. Interference-free communication between GPUs and memory modules is achieved through directional wireless links, while omnidirectional wireless interfaces connect cores in the CPUs with other components in the system. Besides providing low-energy, highbandwidth inter-chip communication, the wireless interconnection scales efficiently with system size to provide high performance across multiple chips. The proposed inter-chip wireless interconnection is evaluated on two system sizes with multiple CPU and multiple GPU chips, along with main memory modules. On a system with $4 \mathrm{CPU}$ and $4 \mathrm{GPU}$ chips, application runtime is sped up by $3.94 \times$, packet energy is reduced by $94.4 \%$, and packet latency is reduced by $58.34 \%$ as compared to baseline system with wired inter-chip interconnection.
\end{abstract}

CCS Concepts: • Computer systems organization $\rightarrow$ Interconnection architectures; Heterogeneous (hybrid) systems; • Networks $\rightarrow$ Network on chip;

Additional Key Words and Phrases: Inter-chip wireless, multi-chip system, heterogeneous architectures

\section{ACM Reference format:}

Sri Harsha Gade, M. Meraj Ahmed, Sujay Deb, and Amlan Ganguly. 2019. Energy Efficient Chip-to-Chip Wireless Interconnection for Heterogeneous Architectures. ACM Trans. Des. Autom. Electron. Syst. 24, 5, Article 55 (July 2019), 27 pages.

https://doi.org/10.1145/3340109

This work was supported in part by the U.S. National Science Foundation (NSF) CAREER Grant under Grant No. CNS1553264 and DST Inspire Fellowship Award by Govt. of India under Grant No. IFA12-ENG-23.

Authors' addresses: S. H. Gade and S. Deb, R\&D Block, Indraprastha Institute of Information Technology Delhi, Okhla Phase III, New Delhi 110020; emails: \{harshag, sdeb\}@iiitd.ac.in; M. M. Ahmed and A. Ganguly, 83 Lomb Memorial Drive, Rochester Institute of Technology, Rochester, New York 14623; emails: \{ma9205, axgeec\}@rit.edu.

Permission to make digital or hard copies of all or part of this work for personal or classroom use is granted without fee provided that copies are not made or distributed for profit or commercial advantage and that copies bear this notice and the full citation on the first page. Copyrights for components of this work owned by others than ACM must be honored. Abstracting with credit is permitted. To copy otherwise, or republish, to post on servers or to redistribute to lists, requires prior specific permission and/or a fee. Request permissions from permissions@acm.org.

(C) 2019 Association for Computing Machinery.

1084-4309/2019/07-ART55 \$15.00

https://doi.org/10.1145/3340109 


\section{INTRODUCTION}

Multichip computing modules with several chips integrated with main memory banks are found in a wide range of platforms from servers to embedded systems. The integrated chips in such systems include processing chips such as multicore CPUs, GPUs, accelerators, or a heterogeneous mix of such chips (e.g., nVIDIA's DGX-1 System [30]) depending on the desired functionality. Application performance in such systems is as highly dependent on efficient interconnection as it is on the efficiency of the processing architectures. Especially, processor-to-memory communication and remote communication between processing chips have a significant impact on the achievable performance. However, inter-chip interconnection in multi-chip systems faces several challenges, viz., (i) exponential increase in memory bandwidth requirements of each individual chip due to the significant ramp up in the number of cores on a single chip is a major concern for performance; (ii) increasing prevalence of memory intensive applications like machine learning and IoT further exacerbates the memory bandwidth challenges; and (iii) the scaling up of number of chips in the multi-chip system poses difficulties for efficient management of shared resources like interconnection and memory subsystem.

Integration of heterogeneous processing architectures further adds to the interconnection performance and resource management challenges due to diverse traffic patterns of different architectures and their interactions. In this work, we primarily focus on CPU-GPU multi-chip systems, which are fast garnering significant interest because of their unique capabilities in handling different workloads. While we discuss the communication bottlenecks in the light of CPU-GPU setups, the limitations remain same for any heterogeneous multi-chip system. Besides processor to memory communication, major bottlenecks for inter-chip communication, even in single CPU-single GPU systems, arise from (i) data communication between host CPU and GPU and (ii) remote communication between the chips (CPU/GPU). The former challenge arises from the way CPU-GPU systems have traditionally been integrated using PCIe switches to connect one or more GPUs to host CPU. While GPUs have access to dedicated VRAM for high-bandwidth memory communication, host communication and data transfer from main memory through PCIe switches severely limits the performance and has high energy consumption. The significantly different access patterns of GPUs and CPUs (high throughput for GPU and low latency for CPU, in general) and interference between both processors compounds the limitations imposed by existing interconnection infrastructure. Unified memory [14] addressing model helps simplify access mechanisms to an extent, but the underlying hardware interconnection limitations still exist.

The latter problem of remote inter-chip communication is especially a limiting factor in systems with multiple CPUs and multiple GPUs. Though single CPU/GPU setups experience limitations, they are not as profound. In multi-chip setups, the interconnection network exhibits very high latency, when a requesting core tries to access remote memory locations from another chip. Multiple GPUs sharing same CPU host further limit available bandwidth as all GPUs have to share the PCIe channels and limit scalability for memory intensive workloads [2,17]. Moreover, each of the heterogeneous system components might operate at different voltages, frequencies and on-chip protocols and will need voltage/frequency or protocol conversion, while communicating with memory modules or other chips in the system. Existing interconnection modalities, based on wired links, for connecting multiple chips fall severely short in providing the desired communication performance requirements of heterogeneous multi-chip systems. Therefore, a prudent, energy-efficient, and scalable interconnection network is required, which can address homogeneous, heterogeneous, and memory integration challenges for different multi-chip environments/systems and provide a unified solution to interconnection issues faced in multi-chip systems. 
Several efforts are underway, from both industry and academia, to address the interconnection challenges for inter-chip communication. In-package memory techniques like three-dimensional (3D) integration provides efficient memory communication; however, they suffer from high thermal density and also do not solve chip-to-chip communication limitations. Inter-chip interconnection solutions like Intel Omni-Path interconnect [5], AMD Infinity fabric [7] in multi-CPU setups, and NVIDIA NVLink [30,31] for multi-GPU setups provide high-bandwidth communication in multi-chip computing systems. However, these solutions address only multi-CPU or multi-GPU setups, whereas we address a similar issue but provide a generic solution that extends to communication between any two chips, either processor or memory.

In this work, we propose to use wireless interconnects to provide an energy-efficient, scalable, and unified solution for interconnecting a heterogeneous multi-chip system with multicore processors, GPUs, and in-package memory modules. Multi gigabits-per-second wireless interconnects through on-chip embedded antennas are a reality in the backdrop of $5 \mathrm{G}$ communication and Internet-of-Things (IoT) [36] and provide the backbone for the proposed chip-to-chip interconnection. It uses a combination of omnidirectional and directional wireless links to provide interference-free communication among all the chips, while meeting the performance requirements of each processor. Using directional dual-band on-chip antennas, we propose a wireless interconnection architecture that establishes direct wireless links between GPUs and memory banks, while simultaneously sustaining non-directional single-hop wireless links between processor tiles in different processor chips. The system wide non-directional links provide low-latency, low-energy communication for CPU-to-memory and CPU-to-CPU/GPU traffic. The dedicated directional wireless links provide sustained, high-bandwidth communication desired by GPU architectures. Furthermore, wireless links, operating at radio frequencies, establish uniform interconnection between chips operating at any voltage/frequency or communication protocols. Each processing chip and memory module in the system is equipped with at least one wireless transceiver to enable inter-chip communication. We propose a Medium Access Control (MAC) and routing protocol suitable for hybrid wireless interconnect fabric and evaluate it in comparison to state-of-the-art and emerging interconnect technologies. The major contributions of this work are as follows:

- A hybrid chip-to-chip wireless interconnection using omnidirectional and directional wireless links for energy efficient communication in heterogeneous multi-chip systems.

- Point-to-point directional wireless links for sustained, high-bandwidth communication between GPUs and main memory modules. System wide omnidirectional wireless infrastructure for low-latency communication between any two chips.

- MAC and routing protocols for the hybrid wireless interconnection to provide interferencefree, high-performance communication in multi-chip systems.

- Detailed evaluation of proposed wireless interconnection at different system setups and wireless links implementations.

The remainder of the article is organized as follows. Section 2 summarizes the state-of-theart and existing works addressing the inter-chip communication challenges in high-performance computing systems. The CPU/GPU multi-chip system and the proposed inter-chip wireless interconnection architecture are described in Section 3. Section 4 provides details of the wireless infrastructure. The communication protocol for providing interference-free, reliable wireless transmission between chip is described in Section 5. The experimental setup and evaluation of the proposed interconnection are provided in Section 6. Section 7 provides a basic view of different communication behaviours that impact extension of proposed hybrid wireless interconnection to any heterogeneous multi-chip architecture. Finally, our work is concluded in Section 8. 


\section{RELATED WORK}

The proliferation of multi-chip computing modules (either on a single die or in a system) and the trend toward heterogeneous computing platforms necessitates efficient and seamless inter-chip interconnection backbone to complement the raw computing power of different architectures. Toward achieving this, there have been several efforts from both industry and academia addressing different aspects of the chip-to-chip communication challenges.

\subsection{State of the Art}

Traditional interconnection modalities, according to International Technology Roadmap for Semiconductors (ITRS) (http://www.itrs2.net/), have not been scaling at the same rate as on-chip interconnects and gate lengths. This implies a huge gap in density and performance of traditional I/O systems and the ever-increasing communication demands of high-performance computing systems. The wiring complexity as number of individual chips increases only further exacerbates the problem due to design challenges, crosstalk, and signal integrity issues. State-of-the-art in-package memory techniques like 2.5D (memory on same substrate or interposer) [41] or 3D (vertical stacking of memory over processing die) [21,24] integration address off-chip memory communication challenges to provide low-latency and high-bandwidth memory access. However, either or both of these techniques suffer from high power/thermal density, limited memory size, and low reliability and yield due to through-silicon vias, thin wafer sizes, layer misalignment, and so on [21, 23, 41]. Additionally, they do not completely eliminate off-chip access and remote chip communication still remains a challenge in multi-chip systems.

To address the inter-chip communication challenges in multi-socket CPU setups, Intel and AMD have introduced Omni-Path Architecture (OPA) [4, 5, 15] and Infinity Fabric (IF) [7], respectively. Intel OPA, described through a series of layers of network stack, provides low-latency, high-bandwidth interconnect fabric between CPU and memory components in large-scale highperformance systems and data centres. AMD IF uses two communication planes, scalable data and scalable control fabrics, that provide coherent interconnection across single chip and multisocket architectures in any topology [37]. NVLink [30, 31], introduced by nVIDIA, addresses interconnection challenges in multi-GPU setups to provide high-bandwidth communication through point-to-point channels between GPUs and CPU (of the order of 5 to 12 times as compared to PCIe). However, these state-of-the-art solutions mostly address only either multi-CPU or multi-GPU setups. Intel's Embedded Multi-Die Interconnect Bridge (EMIB) [8, 25] embeds small silicon bridges and uses microbumps to interconnect multiple dies on a heterogeneous chip package. By avoiding expensive interposer and TSVs, EMIB aims to improve yield as compared to 2.5D technology and interconnect heterogeneous dies (different processing nodes, analog/digital) efficiently. Our work proposes a generic solution that extends to communication between any two chips in single package or multi-chip systems. Furthermore, limitations of electrical links in these interconnections limit the achievable performance to meet the future demands of high-performance computing systems. Toward this end, there are efforts to integrate emerging interconnection technologies for high-bandwidth and low-power chip-to-chip communication.

\subsection{Emerging Interconnects}

Due to the limitations of electical interconnects, various novel interconnect technologies such as photonic [39], inductive or capacitive coupling-based [26], and wireless interconnects [34] are being explored to mitigate the performance issues of conventional I/O-based multi-chip systems. Ultra-low-power photonic interconnects are envisioned to provide chip-to-chip connectivity in multi-chip systems [39]. However, pitch of photonic interconnects, laying out of waveguides, and 
precise control of chip temperature profiles, and so on, pose challenges to their implementation. Moreover, this technology is challenging to integrate with standard CMOS processes, typically requiring a separate photonic device layer with large footprints on the chip. While Reference [33] has proposed wireless inter-chip interconnection for system with in-package memory, it had not considered a heterogeneous multi-chip environment and therefore used only an omnidirectional antenna. In Reference [20], physical layer designs for wirelessly connected multi-chip modules have been proposed for high-performance computing environment. A comparative study for inter- and intra-chip communication using emerging technologies has been done in Reference [16]. While these approaches explore implementing emerging interconnects for multi-chip systems, they do not consider heterogeneous architectures. An interconnection methodology using wireless interconnects to integrate heterogeneous systems involving CPU and GPU cores, but within a single chip, has been proposed in Reference [9]. However, significantly more research needs to be conducted to extend it to overcome the bottlenecks of conventional multi-chip integration methodologies. Also, multi-chip systems involving heterogeneous chips are yet to be investigated for an interconnection that can simultaneously handle CPU and GPU traffic efficiently, and provide a unified interface among heterogeneous system components with low integration overhead. Therefore, in this article, we present a novel wireless interconnection architecture to integrate heterogeneous multi-chip systems with different processing chips and main memory, and compare its performance with state-of-the-art technologies.

\section{WIRELESS INTERCONNECTION ARCHITECTURE FOR HETEROGENEOUS SYSTEMS}

In this section, we discuss the CPU/GPU heterogeneous multi-chip system and the proposed inter-chip wireless network, shown in Figure 3, that provides low-latency, low-energy, and highbandwidth communication between different processing chips and memory modules in a heterogeneous multi-chip architecture. While we discuss the proposed interconnection in the context of CPU/GPU architectures, it is extensible to any heterogeneous system.

\subsection{CPU/GPU Heterogeneous System Architecture}

The CPU/GPU heterogeneous multi-chip system consists of multiple many-core CPUs, multiple GPUs, and main memory banks associated with each chip as shown in Figure 1. The cores in each processor chip are interconnected using a wired intra-chip Network-on-Chip (NoC) topology. The total main memory associated with each processor is composed of multiple chips. The processors access the memory through integrated memory controllers, distributed across the processing chip. The inter-chip interconnection is provided through conventional chip-to-chip buses and PCIe in baseline and wireless network in the proposed architecture.

3.1.1 CPU Intra-chip Topology. Each CPU chip in the system is a many-core processor and is modelled Intel Xeon scalable processor platform [18], as shown in Figure 1(a). It is composed of several cores and corresponding cache hierarchy, interconnected using an intra-chip wired mesh topology. Each processing core is based on X86 architecture, capable of handling two threads. The cache hierarchy has three levels, where L1 and L2 caches are private to the core, while L3 cache is shared among all the cores. Each tile in the mesh network is composed of the CPU core, its private caches (L1 and L2) and a shared L3 cache bank. The tile is fitted with a wired router for interconnecting it with other tiles in the mesh network as shown in Figure 1(a). All the routers within the mesh network are connected using 64-bit wired links. Each CPU chip has its associated main memory connected to it through integrated memory controllers in baseline architecture. The intra-chip topology remains same between both baseline and proposed approaches, except for the 


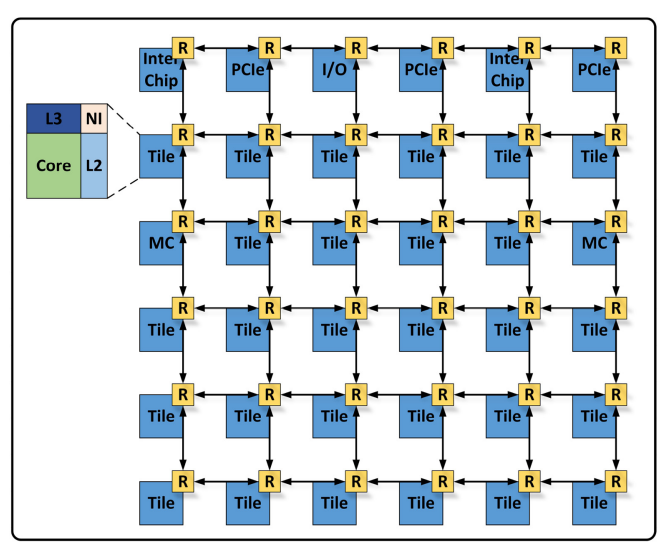

(a) CPU Chip Architecture

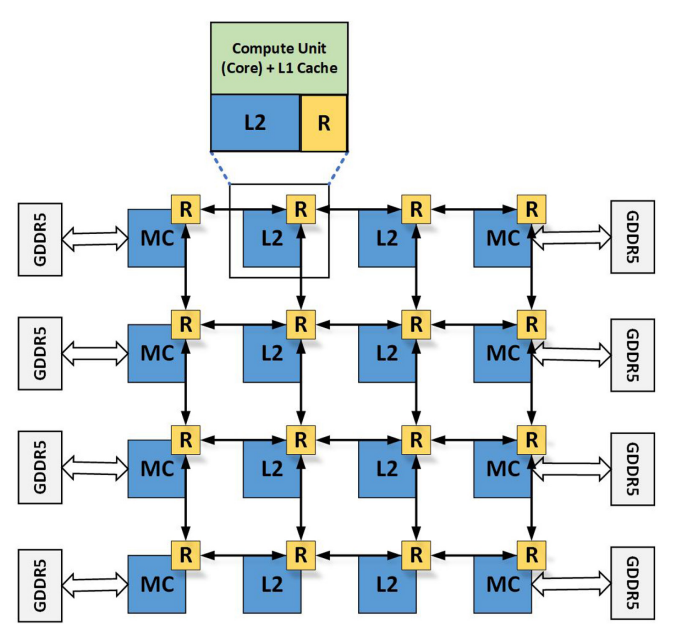

(b) GPU Chip Architecture

Fig. 1. CPU/GPU heterogeneous multi-chip system and intra-chip topologies of different processing chips.

location of memory controllers and I/O peripherals. They are explained in detail in Section 3.1.3 and Section 3.2, respectively.

3.1.2 GPU Intra-chip Topology. The GPU chip in the system is modelled after extant architectures from nVIDIA and AMD, where each compute unit is made up of several hundreds of simple scalar processors. Each compute unit has a private L1 cache, shared by all threads running on the compute unit. In the context of this article, we ignore specialized caches like texture and software managed caches. All the compute units in the architecture share a single L2 cache. The L2 cache is divided into multiple banks and are integrated with memory controllers for accessing off-chip graphic memory. In the processing hierarchy, once a kernel is loaded onto the GPU, the corresponding data is copied from main memory to the graphic memory to provide high-bandwidth memory access. The compute units and L2 cache banks within each GPU are interconnected using a wired mesh topology as shown in Figure 1(a). Each node in the topology is composed of a compute unit, its private cache and a shared L2 cache bank. The inter-router links are made up of 64 bit wires. The integrate memory controllers are distributed across the topology to provide optimal bandwidth and energy consumption in both baseline and proposed architectures.

3.1.3 Baseline Inter-chip Interconnection. In this section, we briefly describe the baseline interchip interconnection for the CPU/GPU heterogeneous system. The baseline inter-chip interconnection follows the traditional approach of networking high-performance multi-socket systems with memory buses, chip-to-chip I/O, and links for CPU and PCIe for GPUs as shown in Figure 2. As described earlier, both CPU and GPU chips communicate with associated main memory through integrated memory controllers. The CPU chips, as shown in Figure 1, each use two memory controllers placed along the first and last columns of the mesh following state-of-the-art systems like Intel Xeon platform [18]. Each memory controller is capable of handling up to three channels and communicates with main memory chips though a bus interconnect. The CPU chips communicate with each other through point-to-point wired links modelled after Intel OPA and corresponding controllers are placed along the first row of the mesh topology. The specifications of both memory and inter-CPU links are provided in Section 6.1. Any CPU chip trying to access remote 


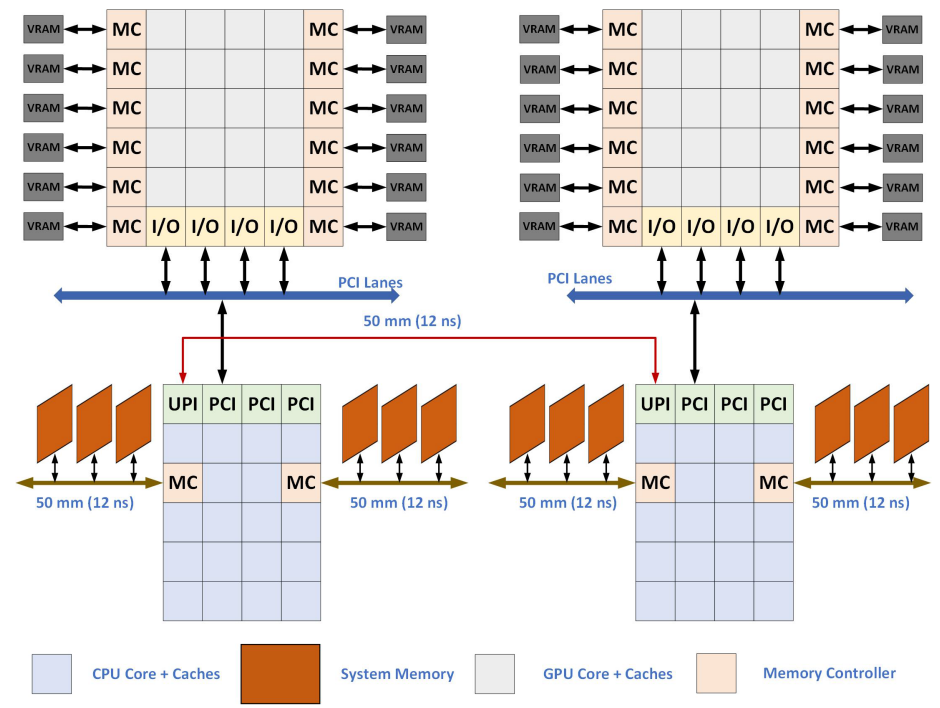

Fig. 2. Baseline inter-chip interconnection for heterogeneous multi-chip systems with buses and PCle links.

memory location (memory associated with another CPU chip) has to forward this request through inter-chip links, which adds considerable performance overheads in baseline architectures.

In the baseline architecture, each GPU connects to a CPU chip through PCIe, through the PCIe controller on the CPU chip as shown in Figure 2. Multiple GPUs can be connected to a single CPU depending on the available number of PCIe lanes on the CPU chip. In our implementations, we assume each GPU chip to be using 16 PCIe lanes and the performance of PCIe links is configured to account for different overheads as highlighted in Reference [22]. Data transfers between main memory and Video RAM (VRAM) present on GPU chips is routed through PCIe controllers and main memory controllers on the CPU chip. Remote communication between two GPUs connected to the same CPU uses the PCIe controllers, while communication between those connected to different CPU chips has to be routed through inter-chip links between the CPU. This remote communication between different chips, either CPU or GPU, adds considerable overhead to the overall performance, when an application is executed across multiple chips. Furthermore, busbased memory interconnection and electrical wire limitations act as congestion points when multiple requests have to be transferred. The proposed inter-chip interconnection overcomes these challenges through high-bandwidth, low-energy wireless network between any two chips.

\subsection{Inter-chip Wireless Network}

The inter-chip wireless network shown in Figure 3, in proposed architecture, is designed to meet the diverse memory access patterns of CPUs and GPUs in performance and energy efficiency, while eliminating the limitations of traditional approaches. CPU cores are traditionally optimized to perform efficiently for serial execution, while GPUs, with thousands of simple scalar threads, work better for highly data parallel applications [29]. Accordingly, CPUs exhibit irregular memory access patterns that require low latency and GPUs have frequent, high-bandwidth memory access pattern to support thousands of cores. To meet these diverse requirements, we implement hybrid wireless network that combines omnidirectional and directional antennas as shown in Figure 3.

The application execution on any single core in multicore CPUs is interspersed by irregular memory access instructions, which stall the execution until the memory request is serviced. 


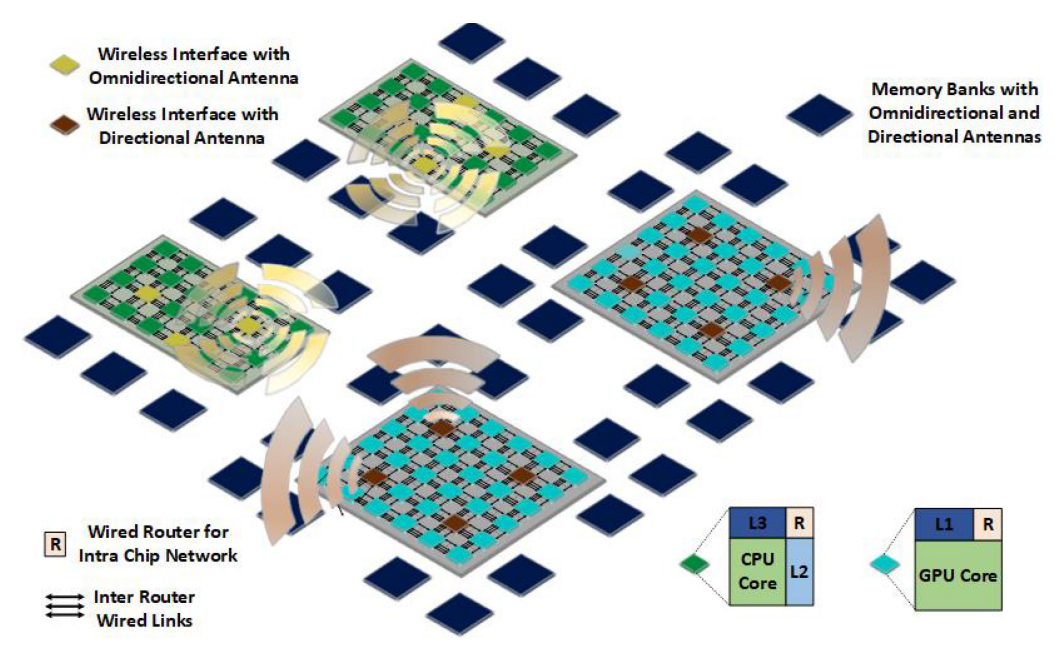

Fig. 3. Chip-to-chip wireless interconnection with directional and omnidirectional wireless links for heterogeneous multi-chip systems.

Though multi-level caches help improve performance, high off-chip memory access latency can significantly impact application performance. In addition, control messages like coherence requests add to overall execution latency, especially when sharer cores are spread across multiple chips. To provide low-latency access to both main memory and remote cores in other CPU/GPU chips, we implement inter-chip wireless network for CPUs using omnidirectional antennas. The omnidirectional wireless network connects each CPU chip with every other processor or memory chip in the system, greatly reducing latency of main memory and remote chip access. This omnidirectional network is implemented on single wireless channel, operating at frequency $f_{\text {omni }}$, and all to and from CPU chips is transmitted over this frequency. In our implementations, we have used $60 \mathrm{GHz}$ frequency for the omnidirectional network. To enable the omnidirectional wireless network for CPU, all its integrated memory controllers, PCIe switches and I/O peripherals are replaced with Wireless Interfaces (WIs) and zigzag antennas. The WI infrastructure and antennas are described in detail in Section 4. The minimal impact of minor distance variations on wireless latency allows a degree of freedom on placement of WIs across the chip. Utilizing this, we optimize the placement of WIs in the mesh network to reduce the average hop count to each the nearest WI and further reduce latency as shown in Figure 3. In addition, our evaluations have shown that four WIs across the chip efficiently cater to the communication needs of the system as opposed to a total of six I/O in baseline system to reduce the area overhead of the network.

Unlike CPU architectures, GPUs hide long memory latency by executing an active threadblock, when current threadblock is stalled due to a memory instruction. They run thousands of threads in parallel and generate regular memory accesses, that have steady, high-bandwidth requirements. To ensure this type of connectivity, we equip GPUs with directional antennas to form point-to-point directional, high-bandwidth wireless links with their associated memory. Similarly to CPUs, the memory controllers on GPUs are replaced with WIs, and their placement is optimized to reduce the intra-chip latency for main memory access as shown in Figure 3. The placement of directional wireless links between each GPU and memory is chosen to provide interference-free, high-bandwidth memory access. As shown in Figure 3, each memory controller on the GPU chip forms directional links with few memory banks. The directional antennas on both memory controller and associated memory banks are aligned to be in the end fire regions of each other to enable seamless 
connectivity. To ensure interference-free and sustained bandwidth over directional wireless network, the radiation regions of the directional antennas are such that (i) the end fire regions of antennas on each link are perfectly aligned with each other and (ii) end fire regions of any two directional links do not have any overlap between them. Hence, the total available bandwidth over the directional wireless network is equal to sum of individual bandwidths of all point-to-point links. It operates at frequency $f_{d i r}$ to avoid interference from omnidirectional network interconnecting GPUs with CPUs over the $f_{\text {omni }}$ channel. In our implementation, we set this frequency to $45 \mathrm{GHz}$, and it is implemented using Planar Log Periodic Antenna (PLPA) [32]. Since the memory modules communicate over both wireless networks, they are quipped with both antenna types and WIs.

The hybrid inter-chip wireless network for heterogeneous architectures caters to the diverse traffic requirements of both CPU and GPU architectures, while providing low-latency, low-energy, and high-bandwidth communication. The directional links provide sustained, high-bandwidth memory access for GPU chips, while omnidirectional network provides low-latency access between any two chips in the system for efficient remote access. The dual channel wireless operation allows simultaneous, interference-free communication to respective memory for both CPU and GPU chips. We have implemented and evaluated the proposed inter-chip interconnection with different modulation techniques and performance capabilities and the necessary wireless infrastructure is described further in detail.

\section{INTER-CHIP WIRELESS INFRASTRUCTURE}

In this section, we describe the wireless infrastructure to implement the proposed hybrid wireless inter-chip interconnection. It uses omnidirectional and directional antennas to provide the hybrid wireless network for different chips in the system. The dual channel wireless transmission require transceivers operating at $f_{o m n i}$ and $f_{d i r}$ frequencies. Furthermore, we implement the transceivers with two different modulation schemes that provide different bandwidth capabilities. The choice of antennas and transceivers is motivated by the necessity to provide high performance with low area and power overheads.

\subsection{Omnidirectional Antenna}

The omnidirectional antenna in the hybrid wireless inter-chip network is implemented using a zigzag antenna as shown in Figure 4(a). It is designed with $10 \mu \mathrm{m}$ trace width, $60 \mu \mathrm{m}$ arm length, and $30^{\circ}$ bend angle. The axial length of the antenna depends on the operating frequency, $f_{\text {omn } i}$, which is designed to operate at $60 \mathrm{GHz}$ in our implementations. The dimensions of the antenna are shown in Figure 4(a). Furthermore, it also has a negligible effect of rotation (relative angle between transmitting and receiving antennas) on received signal strength, making it most suitable for transmission between any two WIs on two remote chips.

\subsection{Directional Antenna}

The directional antenna to form point-to-point wireless links in GPU chips is achieved by use of PLPA [32] as shown in Figure 4(b). PLPA is very popular and widely studied for their ease of manufacturing and their wide-band properties. PLPA, shown in Figure 4(b), is designed to operate at two frequencies for the requirement GPU's communication with main memory and other chips in the systems as described earlier. The antenna is implemented with eight teeth, and the sizes of each teeth increase in logarithmic manner. In our implementation, it is designed to operate at the frequencies of $45 \mathrm{GHz}$ and $60 \mathrm{GHz}$ of the hybrid wireless network. The dimensions of the antenna are shown in Table 1 with the highest dimension being $1.1825 \mathrm{~mm}$ for the desired frequencies. 


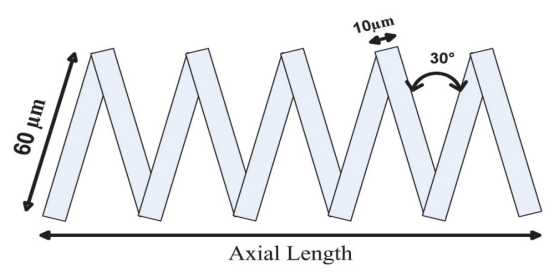

(a) Zigzag Antenna

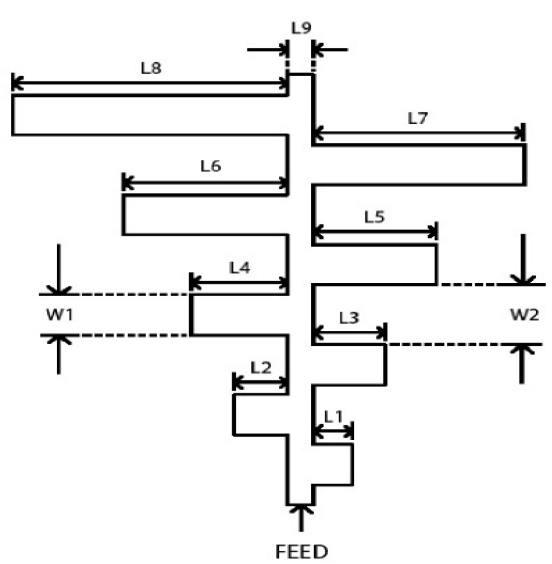

(b) Planar Log Periodic Antenna (PLPA)

Fig. 4. Omnidirectional and directional antennas in the proposed hybrid wireless inter-chip interconnection.

Table 1. Dimensions of PLPA for Directional Wireless Links

\begin{tabular}{|l|c|c|c|c|c|c|c|c|}
\hline Tooth & L1 & L2 & L3 & L4 & L5 & L6 & L7 & L8 \\
\hline Size $(\mathrm{mm})$ & 0.11000 & 0.14019 & 0.17867 & 0.22772 & 0.29023 & 0.36990 & 0.47144 & 0.60085 \\
\hline \multicolumn{2}{|c|}{ Tooth } & \multicolumn{2}{c|}{ L9 } & \multicolumn{2}{c|}{ W1 } & W2 \\
\hline \multicolumn{2}{|c|}{ Size $(\mathrm{mm})$} & \multicolumn{2}{|c|}{05500} & 0.11000 & 0.16500 \\
\hline
\end{tabular}

\subsection{Transceiver Circuitry}

To ensure high throughput and energy efficiency in proposed hybrid wireless inter-chip network, the transceiver circuitry has to provide wide bandwidth as well as low power consumption. The mm-wave frequency band can provide abundant bandwidth without suffering from severe signal degradation, because of the short communication distances. In our implementation, we have studied two different transceiver circuits with modulation schemes, On-Off Keying (OOK) and Orthogonal Frequency Division Multiplexing (OFDM), which provide different bandwidth capabilities.

The transceiver circuit for OOK modulation is shown in Figure 5(a) and has been adopted from Reference [40]. It implements non-coherence OOK modulation scheme and is chosen for its low overhead implementation. The transmitter $\left(T_{X}\right)$ in OOK transceiver consists of an up-conversion mixer and a power amplifier. On the receiver $\left(R_{X}\right)$ side, direct-conversion topology is adopted, consisting of a low noise amplifier, a down-conversion mixer, and a baseband amplifier. An injectionlock voltage-controlled oscillator (VCO) is reused for $T_{X}$ and $R_{X}$ to reduce the area overheads. With both direct-conversion and injection-lock technology, a power-hungry phase-lock loop (PLL) is eliminated. The OOK transceiver design keeps the additional overhead added by WIs, while providing sufficient bandwidth for the proposed interconnection.

The OFDM transceiver implemented in the proposed interconnection is shown in Figure 5(b). It is broadly divided into three parts: (i) data encoding, (ii) implementation of Discrete Fourier Transform (DFT)/inverse DFT, and (iii) cyclic prefix and serial/parallel interfaces. We use M-QAM data encoding scheme to ensure high spectral efficiency with sufficient BER. It encodes $\log _{2} \mathrm{M}$ data bits per symbol into both amplitude and phase of the carrier signal. The FFT module modulates the encoded symbols to respective sub-channel frequency using inverse DFT. The resulting time domain signal is up converted to transmission carrier frequency and amplified using Power Amplifier (PA) 


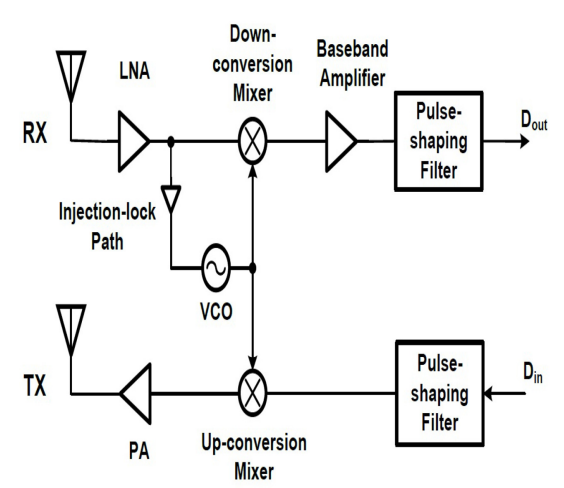

(a) OOK Transceiver

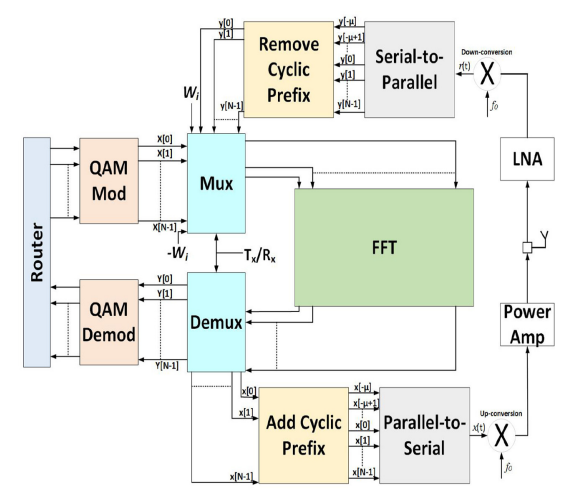

(b) OFDM Transceiver

Fig. 5. Transceiver circuitry of the proposed hybrid wireless inter-chip interconnection.

before transmission. The FFT radix depends on the number of sub-channels chosen. At the demodulator, received signal is down-converted and converted to parallel data streams. Finally, the cyclic prefix bits and symbols are demodulated using DFT operation of FFT module and decoding operations. The serial/parallel interfaces in the transceiver convert data between parallel data streams to time domain sequence. The FFT module performing inverse DFT and DFT operations is reused between transmitter and receiver to reduce area overheads. Since, each of the omnidirectional and directional wireless networks use a single antenna, only the transmitter or receiver is active at any time and so the transceiver has to either modulate or demodulate the signal accordingly. Utilizing this, we share the FFT hardware between modulation and demodulation operations of OFDM. The QAM symbols at modulation form the frequency components of OFDM signal. Inverse DFT at modulator converts these components to time samples and DFT at demodulator converts time samples back to corresponding symbols. The operations differ from each other only by the twiddle factor; $e^{\frac{j 2 \pi i n}{T_{N}}}$ and $e^{\frac{-j 2 \pi i n}{T_{N}}}$ for modulation and demodulation, respectively. Hence, we use the same FFT hardware and provide appropriate set of twiddle factors for each operation to keep the area and power overhead to minimum. The $T_{X} / R_{X}$ control signal chooses the appropriate factor by use of multiplexer and demultiplexer circuits as shown in Figure 5(b).

The performance and overheads of the OFDM modulation depend primarily on number of subchannels, cyclic prefix, and available channel bandwidth, which in turn depend on the channel characteristics. The number of sub-channels in OFDM scheme is determined by coherence bandwidth $\left(B_{C}=1 / T_{m} ; T_{m}\right.$ is maximum delay spread in channel) as $N>>B / B_{C}=B * T_{m}$, where $B$ is the channel bandwidth. Detailed investigations into on-chip wireless channel model in References $[10,11]$ show that delay spread can be as high as $T_{m}=5 \mathrm{~ns}$ and we design the OFDM transceiver to accommodate for this. The number of sub-channels of 256 sufficiently satisfies this criteria in our implementation. Similarly, the number of cyclic prefix bits is determined as $\mu=T_{m} / T_{s}$, where $T_{s}$ is sampling rate of the signal, resulting in 24 cyclic prefix bits. The cyclic prefix bits maintain the orthogonal nature of sub-channels in adverse channel effects at the cost of reduced bandwidth by $N / N+\mu$. Detailed discussion about design criteria for OFDM transceiver for on-chip wireless propagation is available in our previous work [10].

\section{WIRELESS COMMUNICATION PROTOCOL}

In this section, we describe the inter-chip wireless infrastructure management, i.e., medium access control for shared wireless channel and routing algorithm for chip-to-chip communication 


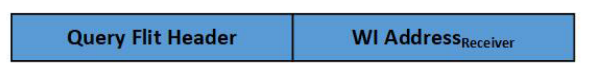

(a) Query Flit

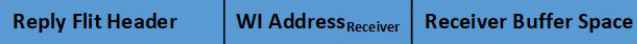

(b) Reply Flit

\section{\begin{tabular}{|l|l|l|}
\hline Transmit Flit Header & List of Receiver Addresses & List of Number of Flits \\
\hline
\end{tabular}}

(c) Transmit Flit

Fig. 6. Flit structure for medium access control and flow control for wireless transmission.

between different processing and memory chips. In the proposed wireless interconnection, we assign each WI across all chips a unique address for medium access control, routing and flow control. The unique WI address, in place of actual destination address, allows for faster address decoding and link establishment, especially in omnidirectional wireless network.

\subsection{Medium Access Control (MAC)}

The dual channel wireless network with omnidirectional and directional links described above enables the simultaneous use of multiple frequency bands and simultaneous transmissions over the point-to-point links. To leverage this, we adopt a hybrid Frequency Division Multiple Access (FDMA) and asynchronous Time Division Multiple Access- (aTDMA) based MAC. The frequency bands around wireless channels $f_{\text {omni }}$ and $f_{d i r}$ are chosen to be non-overlapping to avoid any interference between both wireless networks. The directional wireless network of GPUs and memory establishes point-to-point half duplex links between each memory controller and few respective memory banks. Since no two directional wireless links have overlapping radiation regions, medium access control for directional wireless network is not required. For any memory access on a GPU chip, the request is routed to appropriate memory controller on the chip and then transmitted over the wireless link. While all memory banks linked to this memory controller receive the request, only the memory bank with required data responds to the request over directional link. This provides the dedicated memory access bandwidth necessary for the GPUs without any stalling.

The non-directional antennas operating at $f_{\text {omni }}$ band, deployed in multicore CPUs to enable communication with any other system component, can be oriented in any direction with respect to itself. Due to the potentially large number of WIs across the system in this band, an efficient collision avoidance MAC is necessary. We adopt aTDMA approach with Carrier Sense Multiple Access with Collision Detection (CSMA/CD) for establishing wireless links on-demand. In this MAC mechanism, all WIs sense the omnidirectional wireless channel for any ongoing transmission. The flit structures are shown in Figure 6. When the channel is sensed to be free and a WI has data to send, it transmits a short query flit with only WI address of desired destination as shown in Figure 6(a). As the query flit reaches desired WI, it copies the query flit's content (i.e., the added WI address) and broadcasts a reply flit containing this address and the available buffer space in the destination WI as shown in Figure 6(b). The buffer space field indicates the available free space at the input buffers of the receiving WI. If the available buffer space is not sufficient to store the receiving data, then the transmission is delayed as described in flow control. As the sender WI receives the reply flit, it broadcasts a transmit flit, shown in Figure 6(c), with the number of flits to be transmitted. Both the query flit and transmit flit are received by all WIs, but only the destination WI keeps its receiver ON for the time period mentioned in the transmit flit. Other WIs start a wake-up timer initialized with the value provided in the transmit flit and go to sleep. This further helps maintain energy efficiency with CSMA MAC. After the timer expires, all WIs wake up and go back to sensing mode. 
A collision is detected if there is a failure in receiving reply flit at sender WI within a specific time after the query flit is sent. In case of a collision, the colliding WIs back off from transmitting through wireless channel for time $B_{T}$ given by

$$
B_{T}=r * \eta_{B} * t_{f l i t}
$$

where $\eta_{B}$ is the number of flits a WI must wait before trying to access the channel, $t_{\text {flit }}$ is the time to transmit a flit over the wireless medium and $r$ is a back-off period, which is the duration of back-off before the next transmission is attempted by the WI. This pseudo-random number is generated within a contention window generated by multiplying the back-off factor with a uniform random integer generated by another LFSR implementing exponential back-off. Such back-off, reduces the probability of the WIs from retransmitting at the same time after a collision and reduces probability of collision in future transmissions [19]. When a WI enters the back-off state, it can only receive flits transmitted through the wireless medium. In the back-off period, if a WI receives query flit and transmit flit, then the back-off timer is paused until the wake-up timer expires. The back-off timer restarts counting as the wake-up timer expires.

\subsection{Flow Control and Routing}

In proposed inter-chip wireless interconnection, the end-to-end routing and flow control is dependent on the final destination of the packet. The intra-chip networks in both CPU and GPU chips, based on wired mesh toplogy, use $X Y$ routing algorithm with wormhole flow control for packet transfers. When both source and destination nodes are within the same chip, data is routed simply using the $X Y$ routing algorithm. When the source and destination nodes are from different chips, data from the source is first routed to the appropriate WI using XY routing. The appropriate WI in the source chip depends on the type of packet and type of chip. In CPU chips, it is the nearest WI as each WI can establish communication with any the desired destination, processing or memory chip. In case of GPUs, the choice of WI depends on whether the packet is either memory bound or processor bound. For former, the packet is routed to the WI, which establishes the directional link to the memory bank with required data. For the latter, the packet is routed to the nearest WI for establishing wireless transmission with desired destination processor, which can be CPU or another GPU chip.

Once the packet reaches the appropriate WI, it requests access for the wireless channel with destination being the address of the WI of the packet destination chip. All inter-chip communication, except for GPU to memory communication, is done over omnidirectional network, while the GPU to memory communication uses the directional links. Hence, on GPU and memory chips, the WI makes the decision to use $f_{\text {omni }}$ or $f_{\text {dir }}$ channel depending on the packet destination. As described before, any memory request from/to GPU chip does not require request for channel resources as it is transmitted using directional wireless links over $f_{\text {dir }}$ channel. For all other communication from/to CPU/GPU over $f_{\text {omni }}$ frequency, the channel access mechanism for the same is described in the previous section. While establishing wireless link on-demand in the omnidirectional network, if the buffer space in the reply flit is smaller than the number of flits to be transmitted, the transmitter waits a predefined time before initiating the data transmission. This waiting time is set depending on the average waiting time at any WI for routing packets from its input buffer to output of the router at which the WI is attached. Once channel access is obtained for packet transmission, the packet is transmitted to destination WI. The data from receiving WI is routed to final destination using $X Y$ routing algorithm in destination chip. In the scope of this work, we assume that the flit sizes on all chips is same, though this is not necessary for the proposed interconnection. 
Table 2. Details of CPU/GPU Heterogeneous Systems

\begin{tabular}{|l|l|l|}
\hline Chip & Component & \\
\hline \multirow{4}{*}{ CPU } & Core & x86 cores, 18 cores/chip, 2.5GHz \\
\cline { 2 - 3 } & L1 Cache & 8-way, 64KB cache, 4 cycle, LRU policy, 64 B line, private \\
\cline { 2 - 3 } & L2 Cache & 4-way, 256KB cache, 12 cycle, LRU policy, 64 B line, private \\
\cline { 2 - 3 } & L3 Cache & 16-way, 2MB/core cache, 42 cycle, LRU policy, 64 B line, shared \\
\hline \multirow{3}{*}{ GPU } & Core & Southern Islands, 32 compute units, 64 stream cores/compute unit, 1.5GHz \\
\cline { 2 - 3 } & L1 Cache & 8-way, 32KB cache, 4 cycle, LRU policy, 64 B line, private \\
\cline { 2 - 3 } & L2 Cache & 8-way, 1MB/core cache, 10 cycle, LRU policy, 64 B line, shared \\
\hline
\end{tabular}

Table 3. Details of Intra- and Inter-chip Network

\begin{tabular}{|c|c|}
\hline Component & Configuration \\
\hline \multicolumn{2}{|r|}{ Intra-chip Network } \\
\hline Topology & Wired Mesh for both CPU and GPU \\
\hline Routers & 5 ports (including local port), 64-bit flit, 8 flits buffer per port \\
\hline Links & 64 bits wide, 1 cycle latency \\
\hline \multicolumn{2}{|r|}{ Inter-chip Network } \\
\hline CPU-CPU & \multirow{3}{*}{ Omnidirectional wireless link, $60 \mathrm{GHz}$ carrier } \\
\hline CPU-GPU & \\
\hline CPU-Memory & \\
\hline GPU-Memory & Directional wireless link, $45 \mathrm{GHz}$ carrier \\
\hline OOK Wireless & 16Gbps/link, 1.2pJ/bit \\
\hline OFDM Wireless & 195.32Gbps/link, 0.132pJ/bit \\
\hline
\end{tabular}

\section{EXPERIMENTAL EVALUATION}

In this section, we discuss the experimental setup, architecture of baseline and proposed systems evaluated and performance improvements provided by proposed design.

\subsection{Experimental Setup}

To evaluate the proposed inter-chip wireless architecture for CPU/GPU heterogeneous systems, we use Multi2Sim [35] simulator. It is a cycle accurate heterogeneous architectural simulator, that allows detailed configuration of ISA, memory hierarchy and interconnection topology. The architectural configuration and memory hierarchy of both CPU and GPU chips used in our evaluation is shown in Table 2. The CPU chips are modelled after contemporary high-performance processors like Intel Xeon scalable processor [18] and AMD EPYC [7]. Each CPU chip is composed of 18 cores and each GPU chip is composed of 32 compute units. Each CPU core has private L1 and L2 caches, while L3 cache is shared by all cores. The L3 cache is divided physically into multiple banks (equal to number of cores) and each chip has a total of $36 \mathrm{MB}$ of L3 cache. The CPU core, its private caches and L3 cache segment, along with wired router form a single node as shown in Figure 3. In GPUs, each compute unit has a private L1 cache, while second level cache is shared by all compute units. We evaluate performance and overheads for two systems: (i) $2 \mathrm{CPU}$ and $2 \mathrm{GPU}$ chips (2C-2G) and (ii) 4 CPU and 4 GPU chips (4C-4G).

The configuration of proposed inter-chip interconnection using wireless links for heterogeneous multi-chip systems is shown in Table 3. The intra-chip network, in both baseline and 
Table 4. Total Bandwidth of the Proposed Inter-chip Wireless Interconnection

\begin{tabular}{|l|c|c|c|}
\hline System & Number of Links & OOK & OFDM \\
\hline $2 \mathrm{C}-2 \mathrm{G}$ & 9 & $144 \mathrm{Gbps}$ & $1.72 \mathrm{Tbps}$ \\
\hline $4 \mathrm{C}-4 \mathrm{G}$ & 17 & $272 \mathrm{Gbps}$ & $3.24 \mathrm{Tbps}$ \\
\hline
\end{tabular}

Table 5. CPU and GPU Applications Evaluated

\begin{tabular}{|l|l|}
\hline CPU & $\begin{array}{l}\text { Barnes (BN), BlackScholes (BK), FFT, LU, Ocean (OC), Radiosity (RT), Radix (RX), } \\
\text { Raytrace (RY), Swaptions (SW), X264 }\end{array}$ \\
\hline \multirow{2}{*}{ GPU } & $\begin{array}{l}\text { Binary Search (BS), Back Propagation (BP), Convolution (CL), DCT, Eigen Value (EV), } \\
\text { Fast Walsh (FW), Histogram (HG), Matrix Multiplication (MM), Nearest Neighbour } \\
\text { (NN), Quasi Random (QR), Radix Sort (RS), Stream Cluster (SC) }\end{array}$ \\
\hline
\end{tabular}

proposed architectures, is implemented using wired mesh topology. The router and link configuration are shown in Table 3. The intra-chip routers operate at the same frequency as that of the cores in respective processing chips. The inter-chip interconnection for proposed architecture is implemented using hybrid wireless design with omnidirectional and directional links. The communication between a CPU and all other system chips viz., GPU, CPU and main memory is transmitted using an omnidirectional wireless link operating at $60 \mathrm{GHz}$ carrier frequency. The GPU to main memory traffic is transmitted over directional point-to-point wireless links implemented using PLPA with $45 \mathrm{GHz}$ carrier frequency. Each chip is fitted with wireless interfaces for all inter-chip communication as described in previous sections. The wireless links are implemented using two different modulation techniques, OOK and OFDM as described. The specifications of both designs is provided in Table 3 . The OOK modulation provides a bandwidth of $16 \mathrm{Gbps}$ per link with $1.2 \mathrm{pJ} /$ bit energy consumption, while each OFDM wireless link provides 195.32Gbps bandwidth at $0.132 \mathrm{pJ} /$ bit energy consumption. The $2 \mathrm{C}-2 \mathrm{G}$ and $4 \mathrm{C}-4 \mathrm{G}$ systems, evaluated in this work, have a total of 9 and 17 wireless links operating simultaneously and the total inter-chip bandwidth provided by prooposed interconnection is shown in Table 4. As observed, the proposed inter-chip wireless interconnection provides multiple terabits per second bandwidth using OFDM links. Prudent link, antenna and transceiver designs have the potential to provide significantly higher bandwidths desired by high-performance systems.

The efficiency of proposed inter-chip interconnection is evaluated by simulating different CPU and GPU applications from PARSEC [3], SPLASH2 [38], AMD APP SDK [1], and Rodinia [6] benchmark suites, shown in Table 5. Application scenarios are chosen to be CPU only, GPU only, and concurrent execution of both CPU and GPU applications to sufficiently stress the system. In all application scenarios, the problem sizes are chosen such that all CPU cores or GPU cores or both are active for the entire duration of the application execution. This is to ensure that the system is properly stressed and we capture contention for off-chip resources also. We evaluate network energy, network latency and application runtime of proposed topology and compare it against those of baseline system.

\subsection{Hybrid Wireless Interconnection}

In this section, we briefly evaluate the motivation for implementing hybrid wireless interconnection with directional and omnidirectional antennas. Figure 7 shows the improvements provided by hybrid wireless interconnection as compared with inter-chip wireless network with only 


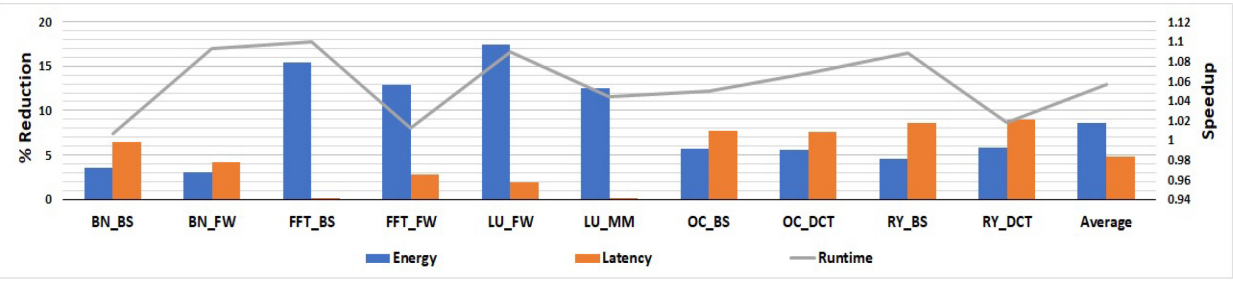

Fig. 7. Comparison between hybrid wireless interconnection and omnidirectional only wireless network.

omnidirectional antennas. The results are obtained using OOK modulation described in Table 3 and the system with only omnidirectional links is implemented as a dual channel wireless network with $45 \mathrm{GHz}$ and $60 \mathrm{GHz}$ carriers. As can be observed, the hybrid wireless interconnection provides better energy savings upto $20 \%$ and runtime speedup upto $1.1 \times$ as compared to using only omnidirectional antennas. This improvement is primarily due to reduced contention for wireless channel and interference-free communication for GPUs. While a system with only directional wireless links may reduce interference for inter-chip interconnection and provide higher overall throughput, it poses implementation challenges. It is infeasible to implement several directional wireless links between all chips in the system without any overlap between their radiation regions. Furthermore, the directional antennas incur higher area overheads as compared to omnidirectional antennas as detailed in Section 6.5.

\subsection{Network Energy}

One of the major advantages of wireless interconnects is their energy efficiency over wired links for long distance communication. Figure 8 and Figure 9 show the saving in average packet energy in the network using inter-chip wireless interconnection as compared to baseline wired interconnection for both system sizes evaluated. The energy results are shown for both OOK- and OFDM-based implementations. The results also show savings in routers and links separately to clearly highlight the contribution of wireless links over wired links. The interconnect energy includes both on-chip and off-chip packet communication, along with serialization, antenna and analog component overheads of the wireless interface. These overheads are discussed in detail in Section 6.5. As observed from Figure 8, with single CPU and single GPU application running simultaneously, OOK- and OFDM-based inter-chip wireless network saves $92.08 \%$ and $934.09 \%$ of average packet energy, respectively, as compared to baseline interconnection on a system with two CPU and two GPU chips. Similarly, on system with four CPU and four GPU chips from Figure 9, OOK- and OFDM-based inter-chip wireless interconnection saves packet energy of $92.94 \%$ and $93.46 \%$, respectively, as compared to baseline. Furthermore, the improvement in network energy is higher when multiple applications are running simultaneously as opposed to single application, showing the scalability of wireless interconnection under high stress.

Figure 10 shows the contribution of different on-chip and off-chip components to the overall network energy consumption under different application scenarios. The energy breakdown results are averaged over all benchmarks in each application scenario; CPU only (C), GPU only $(\mathrm{G})$, and $\mathrm{CPU}+\mathrm{GPU}(\mathrm{CG})$ for baseline, OOK-based, and OFDM-based wireless interconnections. In Figure 10, tr $_{O N-C P U}$ and tr $_{O N-G P U}$ represent router energy within CPU and GPU chips, respectively, while $L i n k_{O N-C P U}$ and $L i n k_{O N-G P U}$ represent link energy within CPU and GPU chips, respectively. The off-chip network energy consumption is broken down in CPU-to-CPU energy $\left(\operatorname{Rtr}_{C 2 C}\right.$ and $\left.\operatorname{Link}_{C 2 C}\right)$, CPU-to-Memory energy $\left(R \operatorname{tr}_{C 2 M}\right.$ and $\left.\operatorname{Link}_{C 2 M}\right)$, and GPU-to-Any energy $\left(\operatorname{Rtr}_{G 2 X}\right.$ and $\left.\operatorname{Link}_{G 2 X}\right)$ components. In the baseline system, the off-chip wired links 


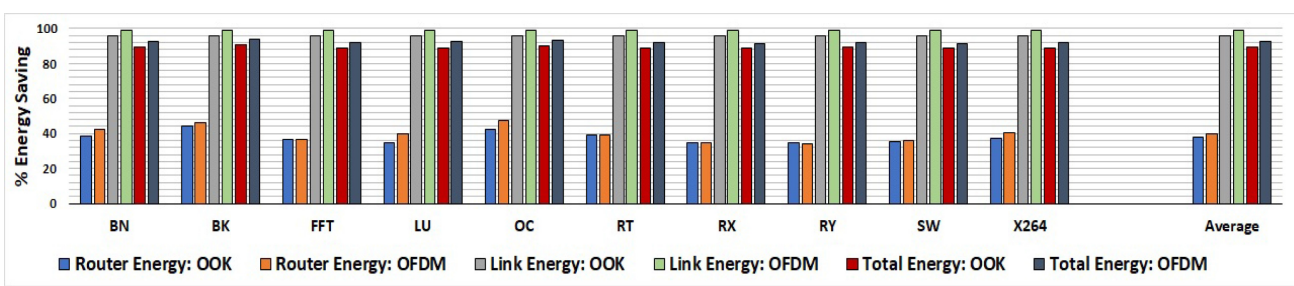

(a) CPU Only Applications

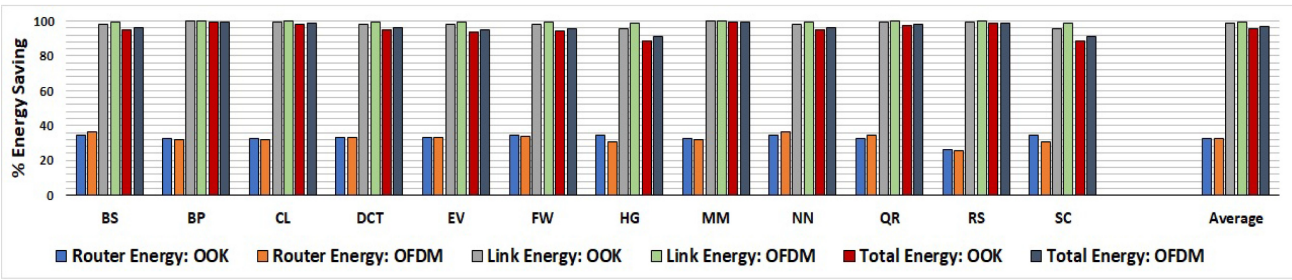

(b) GPU Only Applications

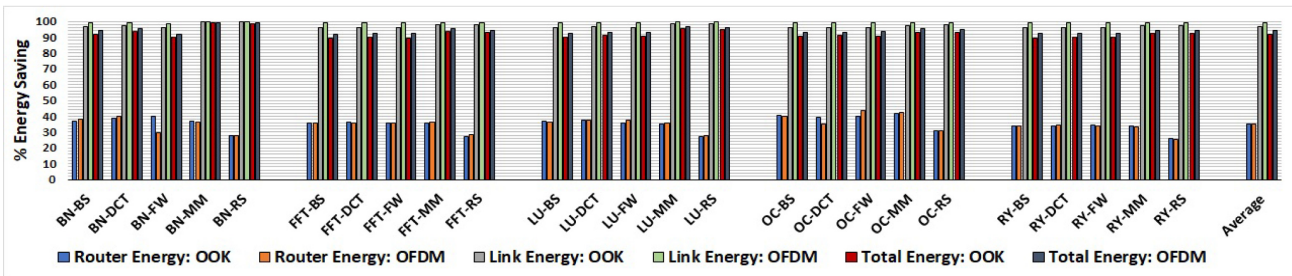

(c) Single CPU and Single GPU Application running simultaneously

Fig. 8. Average packet energy saving achieved by wireless inter-chip interconnection against traditional wired network on system with two CPU and two GPU chips.

contribute to most of the network energy consumption, accounting for $92.39 \%$ and $94.03 \%$ of total network energy on $2 \mathrm{C}-2 \mathrm{G}$ and $4 \mathrm{C}-4 \mathrm{G}$ systems, respectively, across different application scenarios. In baseline architecture, all GPU off-chip communication happens over PCIe bus, while CPU off-chip communication happens over memory and chip-to-chip buses, respectively. In comparison, the OOK-based wireless inter-chip interconnection reduces the contribution of off-chip link energy to $30.49 \%$ and $33.80 \%$ of total network energy on $2 \mathrm{C}-2 \mathrm{G}$ and $4 \mathrm{C}-4 \mathrm{G}$ systems, respectively. In the wireless inter-chip interconnection, $\operatorname{Link}_{C 2 C}$ represents all communication over the omnidirectional network, while $\operatorname{Link}_{G 2 X}$ represents directional wireless network communication. The off-chip link energy consumption is further reduced to $10.85 \%$ and $10.51 \%$ of total network energy using OFDM-based wireless links because of their better per bit energy efficiency.

As observed from Figure 10, the network energy saving achieved with wireless inter-chip interconnection clearly highlights the efficiency of wireless links in optimizing energy consumption for off-chip communication as compared to PICe and bus-based interconnection of baseline architecture. Using a hybrid combination of such energy efficient wireless links, the proposed inter-chip interconnection reduces link energy by $97.39 \%$ and $99.16 \%$ with OOK and OFDM modulation, respectively, on the 4C-4G system as seen from Figure 9. The wireless links, besides exhibiting better energy efficiency as compared to wired links, also reduce application runtime and overall active period of both on-chip and off-chip network components. Combined with lower average hop count to reach off-chip switches, the wireless interconnection also saves router energy by $38.21 \%$ on the 


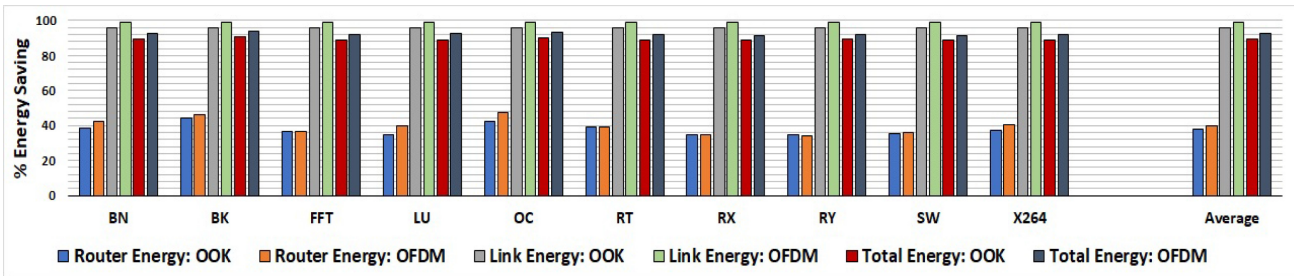

(a) CPU Only Applications

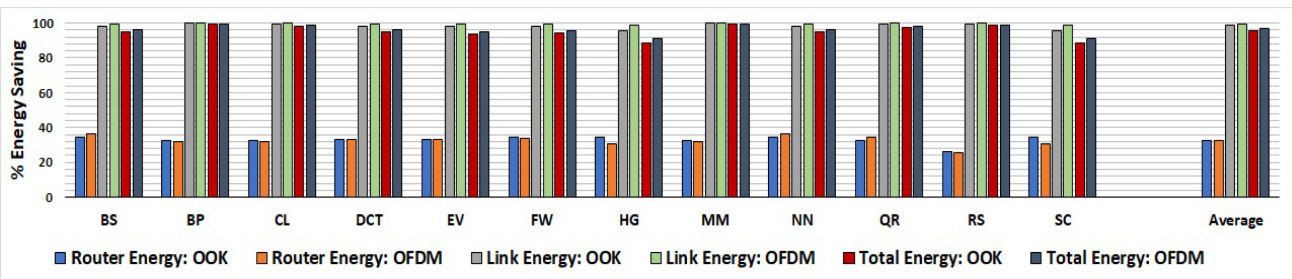

(b) GPU Only Applications

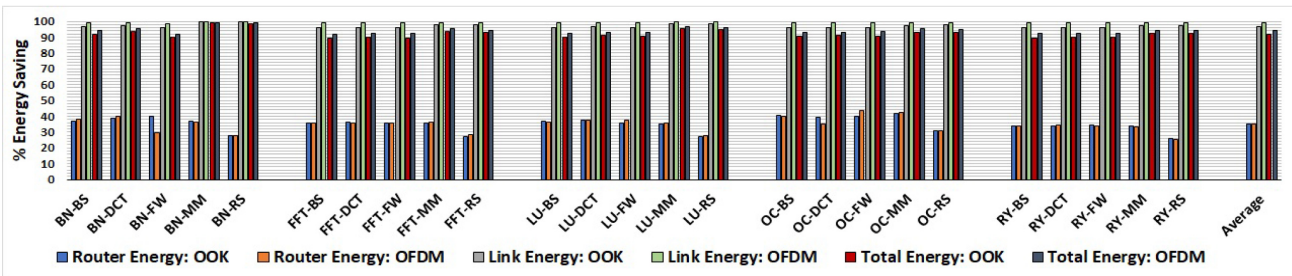

(c) Single CPU and Single GPU Application running simultaneously

Fig. 9. Average packet energy saving achieved by wireless inter-chip interconnection against traditional wired network on system with four CPU and four GPU chips.

$4 \mathrm{C}-4 \mathrm{G}$ system, further improving the network energy efficiency as compared to wired interconnection of baseline architecture.

\subsection{Performance Evaluation}

The performance of the proposed inter-chip wireless interconnection is evaluated in terms of average packet latency of the network and total application runtime.

6.4.1 Network Latency. One of the major challenges for performance in many-core architectures is off-chip memory access latency and bandwidth. This is further exacerbated in multi-chip systems, especially for accessing remote chip and memory elements. Wireless links exhibit linear latency characteristics over large distances and provide high bandwidth making them ideal for overcoming inter-chip performance bottlenecks. Figure 11 and Figure 12 show the reduction in average packet latency using inter-chip wireless interconnection in comparison with baseline system using wired bus and PCIe links on $2 \mathrm{C}-2 \mathrm{G}$ and $4 \mathrm{C}-4 \mathrm{G}$ systems, respectively. On average, across different concurrent application scenarios, the packet latency is reduced by $57.19 \%$ and $57.32 \%$ for $2 \mathrm{C}-2 \mathrm{G}$ and $4 \mathrm{C}-4 \mathrm{G}$ systems, respectively, using OOK-based inter-chip wireless network as compared to baseline interconnection. Using the OFDM-based wireless links, the average packet latency is reduced by $57.59 \%$ and $58.34 \%$ for $2 \mathrm{C}-2 \mathrm{G}$ and $4 \mathrm{C}-4 \mathrm{G}$ systems, respectively. The network performance scales with multiple applications running simultaneously as observed. Furthermore, 


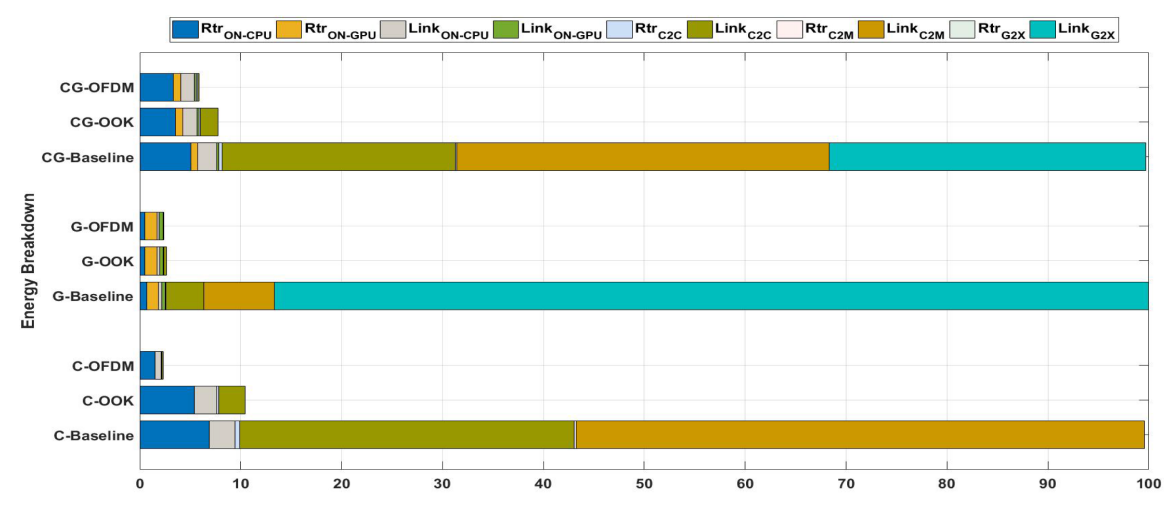

(a) 2C-2G System

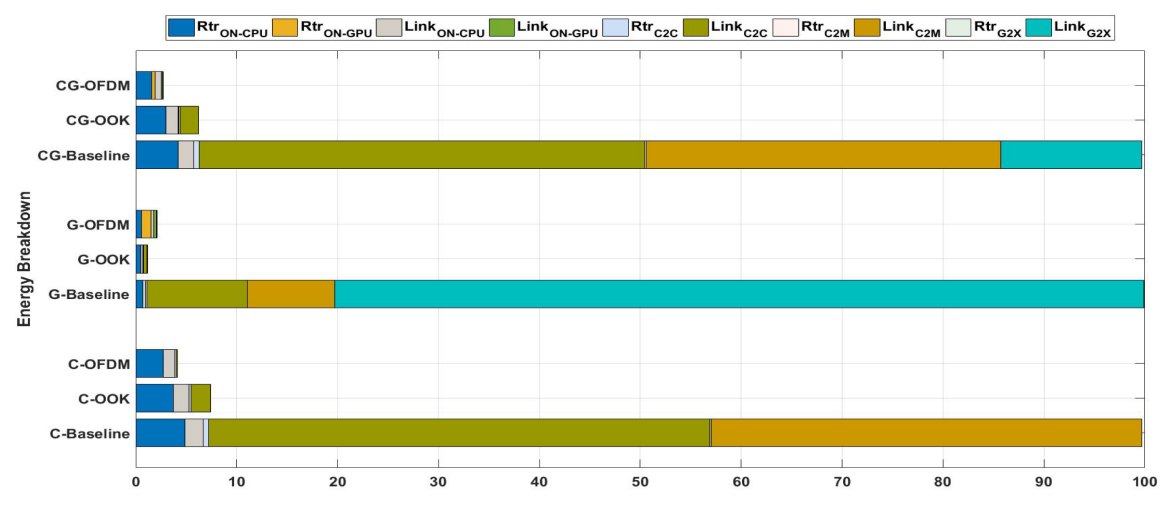

(b) 4C-4G System

Fig. 10. Breakdown of network energy consumption on baseline and wireless inter-chip interconnection for different application scenarios.

wireless links with their superior performance across long distances avoid the limitation of keeping off-chip I/O components along chip edges as is traditionally done. This provides freedom to designers to optimally place the off-chip switches across the chip to optimize intra-chip performance for off-chip communication and also reduce on-chip average packet latency.

To further highlight the efficiency of wireless links in optimizing off-chip communication performance, Figure 13 shows the breakdown of total packet latency into different latency components under different application scenarios for all evaluated architectures. Similarly to energy breakdown, the latency breakdown is also shown for each application scenario for baseline, OOKbased, and OFDM-based wireless interconnections. Latency $y_{O N}$ in Figure 13 represents the average packet latency within chip components (both CPU and GPU), while Latency ${ }_{C 2 C}$, Latency ${ }_{C 2 M}$, and Latency $_{G 2 X}$ represent the off-chip latency for CPU-to-CPU, CPU-to-Memory and GPU-to-Any communication, respectively. Figure 13 shows that the off-chip communication latency contributes to a significant portion of the overall packet latency in baseline architecture under any application scenario. On average, off-chip communication latency accounts for $42.66 \%$ of total latency under different application scenarios on 2C-2G system as seen from Figure 13(a). The major portion of 


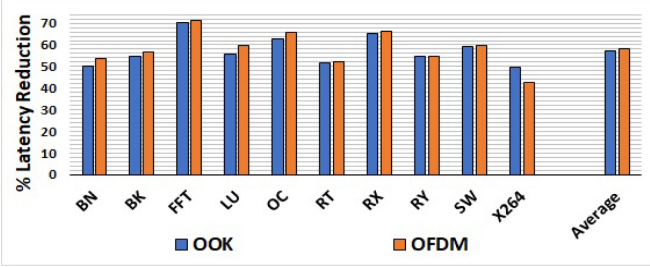

(a) CPU Only Applications

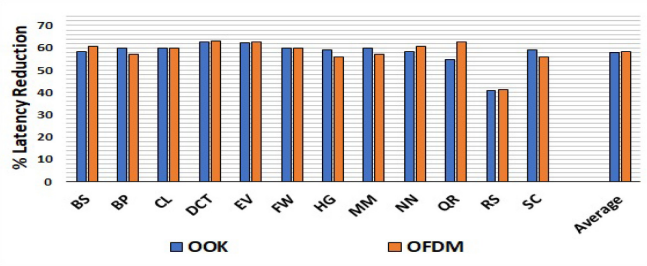

(b) GPU Only Applications

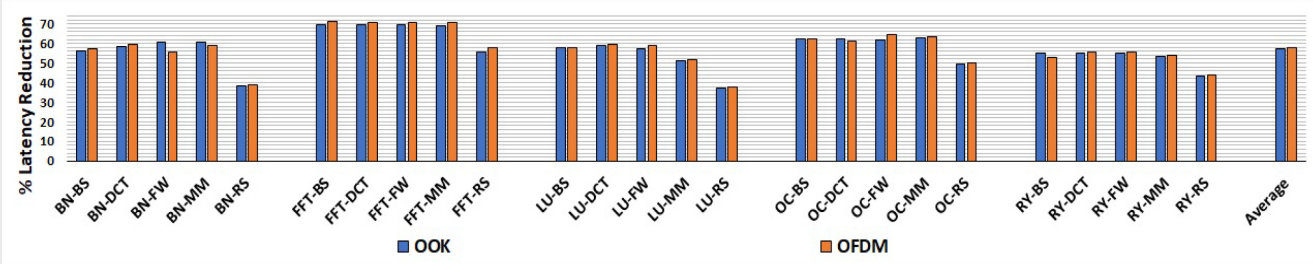

(c) Single CPU and Single GPU Application running simultaneously

Fig. 11. Reduction in average packet latency using wireless inter-chip interconnection against traditional wired network on system with two CPU and two GPU chips.

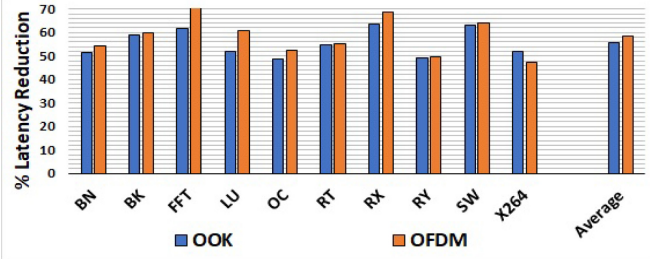

(a) CPU Only Applications

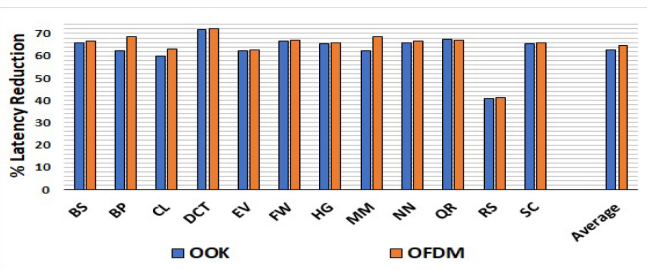

(b) GPU Only Applications

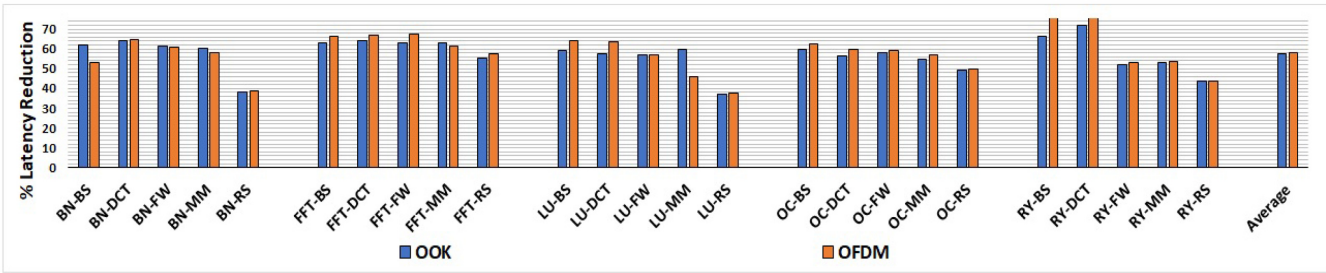

(c) Single CPU and Single GPU Application running simultaneously

Fig. 12. Reduction in average packet latency using wireless inter-chip interconnection against traditional wired network on system with four CPU and four GPU chips.

off-chip communication latency is contributed by main memory communication to service all memory requests from CPU/GPU. The contribution of off-chip latency increases to $66.05 \%$ on the $4 \mathrm{C}-4 \mathrm{G}$ system as seen from Figure $13(\mathrm{~b})$. Especially, the Latency ${ }_{\mathrm{C} 2 \mathrm{C}}$ increases from $\approx 4 \%$ on $2 \mathrm{C}-2 \mathrm{G}$ system to $\approx 23 \%$ on $4 \mathrm{C}-4 \mathrm{G}$ system clearly highlighting the limitations of wired inter-chip interconnection in servicing remote requests across different chips, along with main memory communication.

In contrast, the OOK- and OFDM-based wireless inter-chip interconnection reduces the off-chip communication latency to $5.56 \%$ and $3.58 \%$ of total latency, respectively, on $2 \mathrm{C}-2 \mathrm{G}$ system as seen 


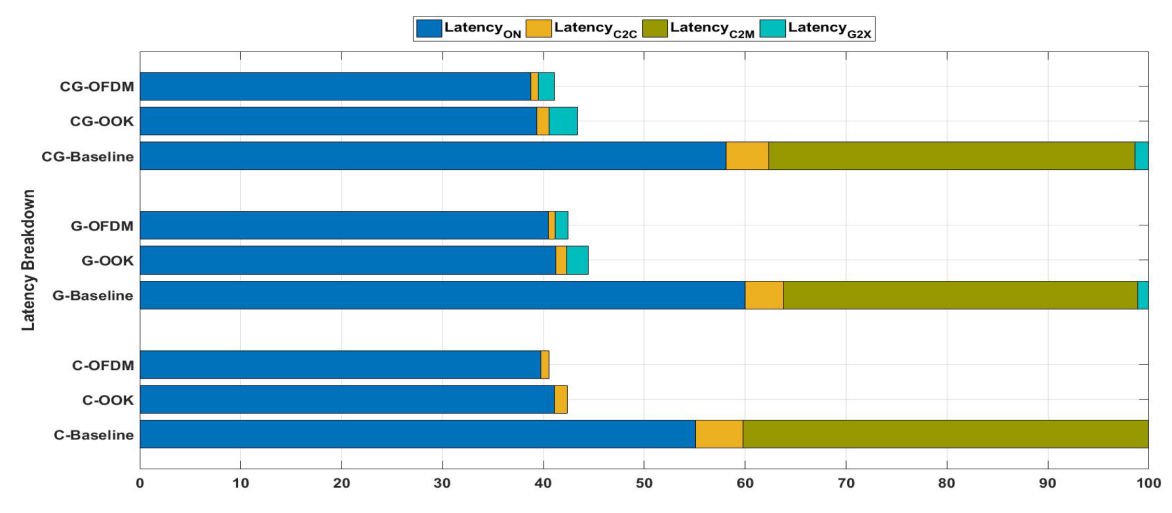

(a) 2C-2G System

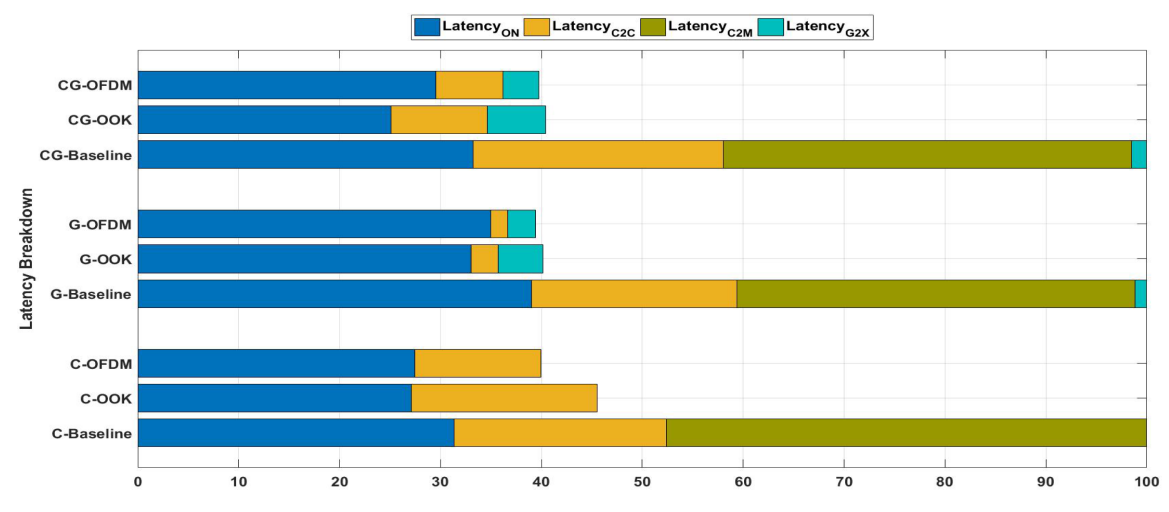

(b) 4C-4G System

Fig. 13. Breakdown of average packet latency on baseline and wireless inter-chip interconnection for different application scenarios.

from Figure 13(a). In the figure, Latency ${ }_{C 2 C}$ represents omnidirectional wireless network latency and Latency $y_{G X}$ represents directional network latency. On $4 \mathrm{C}-4 \mathrm{G}$ system from Figure 13(b), the OOK- and OFDM-based off-chip wireless links contribute to $8.38 \%$ and $5.08 \%$ of total network latency under different application scenarios. The slight increase in the off-chip communication latency on $4 \mathrm{C}-4 \mathrm{G}$ system is attributed to the increased waiting latency at each transceiver for accessing omnidirectional channel. From Figure 13, the low-latency communication of wireless links across long distances clearly improves off-chip communication significantly in accessing both main memory and remote chips. This is further aided by the hybrid interconnection, which minimizes waiting and channel access latencies for GPU off-chip communication and improves overall off-chip communication performance. The off-chip wireless communication latency, as number of chips in the system increases, can be optimized by exploring and adopting efficient channel sharing [13] and channel access mechanisms [12] to reduce waiting time at each transceiver. Furthermore, the on-chip communication latency can also be improved by optimizing the placement of I/O and transceivers across the chip as opposed to chip edges as is the case in wired inter-chip interconnection architecture. 


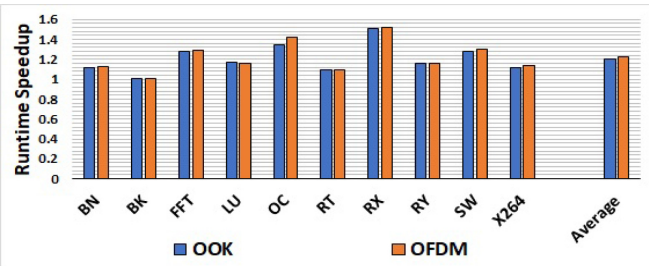

(a) CPU Only Applications

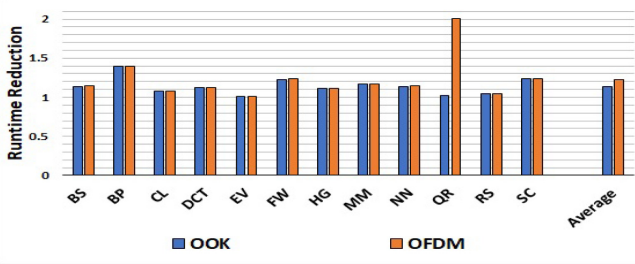

(b) GPU Only Applications

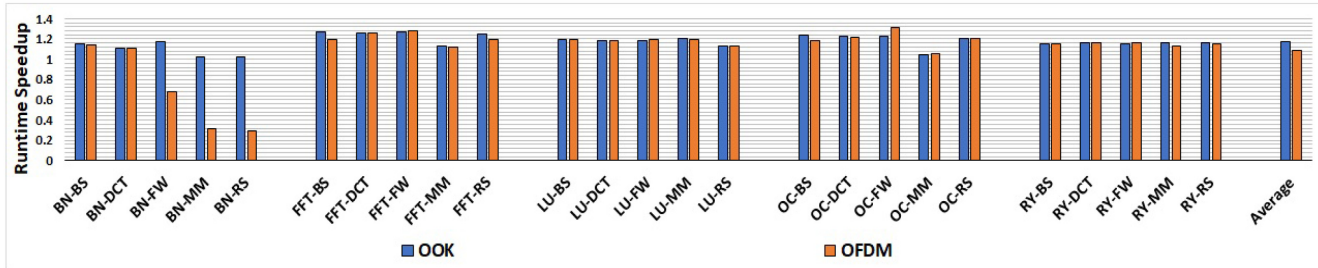

(c) Single CPU and Single GPU Application running simultaneously

Fig. 14. Application runtime speedup using wireless inter-chip interconnection against traditional wired network on system with two CPU and two GPU chips.

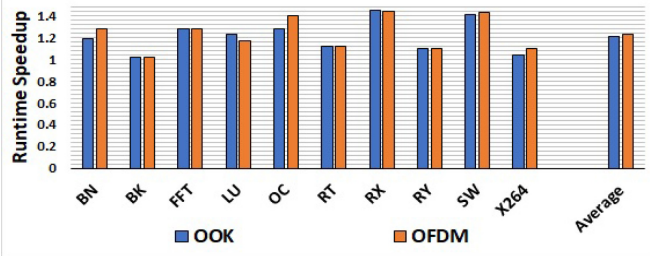

(a) CPU Only Applications

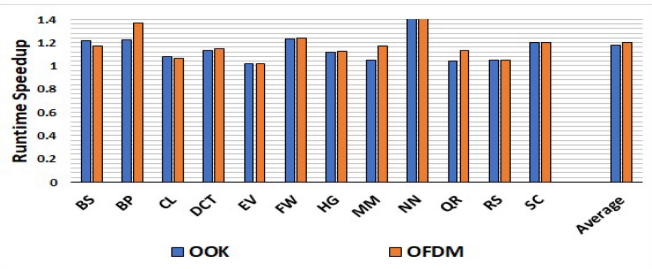

(b) GPU Only Applications

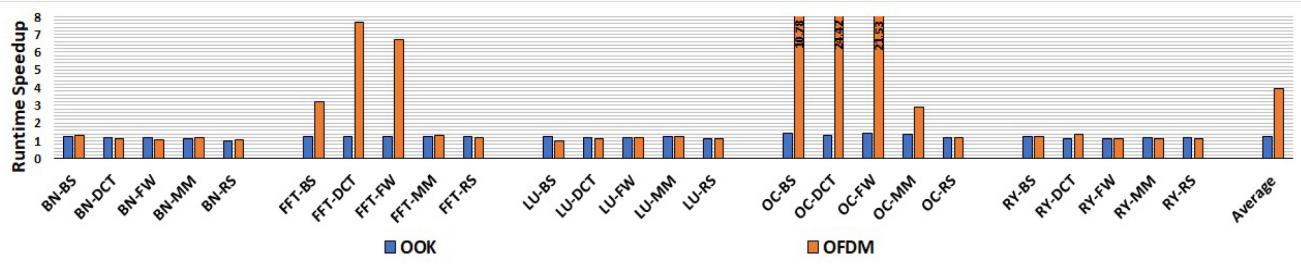

(c) Single CPU and Single GPU Application running simultaneously

Fig. 15. Application runtime speedup using wireless inter-chip interconnection against traditional wired network on system with four CPU and four GPU chips.

6.4.2 Application Runtime. The application runtime improvements using wireless interconnection over baseline are quantified by speedup in execution time for completing application contexts (both CPU threads and GPU kernel (if present)). An application is composed of both computation and memory instructions and speedup is dependent not only on number of memory accesses, but also on distribution of memory accesses throughout the application execution. The application speedup with wireless interconnection over wired interconnection for CPU, GPU, and CPU+GPU applications in both system sizes is shown in Figure 14 and Figure 15. Figures 14(a) and 15(a) and Figures 14(b) and 15(b) show runtime speedup when only either a CPU or GPU 
Table 6. Area and Power Overheads of Inter-chip Wireless Interconnection

\begin{tabular}{|c|c|c|c|c|c|}
\hline Component & Wired Router & OOK WI & OFDM WI & Zigzag Antenna & PLPA \\
\hline Area $\left(\mathrm{mm}^{2}\right)$ & $7.67 \times 10^{-3}$ & 0.30 & 0.15 & 0.022 & 1.33 \\
\hline Power $(\mathrm{mW})$ & 4.46 & 28 & 25.825 & - & - \\
\hline
\end{tabular}

application is running on the system. In such scenarios, the speedup in application is solely due to faster access to off-chip memory and remote locations in other chip components. In case of GPU only application scenarios, a section of application runs on CPU, which handles mainly control tasks, while computation is performed by GPU kernel. Even with baseline system using dedicated memory buses for each memory controller, wireless inter-chip interconnection using OFDM wireless links improves application runtime by $1.22 \times$ and $1.24 \times$ in CPU only application scenarios on $2 \mathrm{C}-2 \mathrm{G}$ and $4 \mathrm{C}-4 \mathrm{G}$ systems, respectively. This clearly shows the performance improvements provided by inter-chip wireless interconnection, even with all CPU chips sharing single channel resources. This is due to significantly high bandwidth and low latency provided wireless links as compared to off-chip buses. In GPU only application scenarios, Figure 14(b) and Figure 15(b) show that the runtime is sped up by $1.10 \times$ using OOK-based wireless links as compared to PCIe-based interconnection. With OFDM-based wireless links, the runtime is further improved by $1.22 \times$ and $1.12 \times$ on both systems, respectively. Even when the system is stressed with concurrent CPU and GPU applications and channel resources being shared, application runtime is improved by $1.17 \times$ and $1.23 \times$ for $2 \mathrm{C}-2 \mathrm{G}$ and $4 \mathrm{C}-4 \mathrm{G}$ systems, respectively, using OOK-based wireless links as compared to baseline interconnection. Using OFDM wireless links, the application runtime is further improved to $1.18 \times$ and $3.94 \times$ on $2 \mathrm{C}-2 \mathrm{G}$ and $4 \mathrm{C}-4 \mathrm{G}$ systems, respectively, in comparison with baseline interconnection as shown in Figure 14(c) and Figure 15(c). The improvements in network latency and application runtime clearly show the potential of hybrid wireless inter-chip interconnection in providing efficient communication performance in heterogeneous multi-chip systems. The performance can be further improved by designing suitable protocol infrastructure like scheduling algorithms, memory access mechanisms that compliment wireless interconnection.

\subsection{Overhead Analysis}

In this section, we evaluate the area and power overheads required to implement the proposed inter-chip wireless interconnection. All network components are synthesized at $45 \mathrm{~nm}$. Table 6 details the overheads of both intra- and inter-chip networks of the proposed interconnection. The wired components of the intra-chip network in both CPU and GPU are designed and synthesized in Synopsys Design Compiler. Each wired router is composed of five ports, including the local port, and is input buffered with 8 -flit wide buffers. It occupies a total area of $7.67 \times 10^{-3} \mathrm{~mm}^{2}$ including all the components. The proposed inter-chip wireless interconnection replaces all $\mathrm{I} / \mathrm{O}$ controllers in baseline system with wireless interfaces for inter-chip communication. As seen from Figure 2 and Figure 3, it replaces the six wired I/O (including memory controllers) in CPU chips and eight I/O in GPU chips of baseline system with four WIs and antennas each. The WI area overhead is the total area consumed by modulator/demodulator, power amplifier, and LNA circuits. The circuitry for OOK modulation requires a total area of $0.16 \mathrm{~mm}^{2}$, while OFDM circuit with 256 sub-channels occupies an area overhead of $0.092 \mathrm{~mm}^{2}$ for each transceiver. The power amplifier and INA circuits in both OOK and OFDM WIs add an area overhead of $0.14 \mathrm{~mm}^{2}$. Hence, the total area overhead for each WI is $0.30 \mathrm{~mm}^{2}$ and $0.15 \mathrm{~mm}^{2}$ for OOK and OFDM modulations, respectively. In addition, the omnidirectional zigzag antenna is $0.38 \mathrm{~mm}$ long and $58 \mu \mathrm{m}$ wide (area of $0.022 \mathrm{~mm}^{2}$ ), while PLPA for directional links has an area of $1.33 \mathrm{~mm}^{2}$. 
Each wired router in the intra-chip network consumes total dynamic and static power of $4.46 \mathrm{~mW}$ and $6.44 \mathrm{~mW}$, respectively. In WI, the power amplifier and LNA contribute to the majority of its power consumption. Together, they both consume $19 \mathrm{~mW}$ of power. The circuitry for OOK modulation consumes $9 \mathrm{~mW}$, while OFDM with 256 sub-channels has a power overhead of $6.825 \mathrm{~mW}$. In total, each WI consumes $28 \mathrm{~mW}$ and $25.825 \mathrm{~mW}$ for OOK and OFDM modulation, respectively. The network energy results presented in the previous section accounts for all WI overheads at each WI. These power overheads, especially static and idle state power consumption, can be minimized by low-power techniques [27, 28] to further reduce the network energy consumption.

\section{EXTENSION TO ANY HETEROGENEOUS ARCHITECTURE}

The proposed hybrid inter-chip wireless interconnection shows tremendous improvements in performance and energy efficiency for heterogeneous multi-chip architectures. While the interconnection design has been discussed in the context of CPU/GPU multi-chip systems, it is extensible to any heterogeneous multi-chip architecture. By abstracting the major characteristics and requirements of all inter-chip communication, a prudent and efficient hybrid wireless interconnection can be designed for a heterogeneous system with any number of different architectures.

A typical heterogeneous multi-chip system consists of CPU acting as master with other application specific hardware for improving performance and/or efficiency. Besides communication with $\mathrm{CPU}$ and main memory, the important off-chip and inter-chip communication behaviours that impact the design of wireless interconnection are as follows:

- Computation/Memory Boundedness: One of the basic and important constraints impacting off-chip interconnection design is the memory or computation boundedness of the applications running on specialized hardware. While computationally intensive applications are less impacted by inter-chip interconnection, memory intensive applications require efficient interconnection architecture. Especially with rise of memory bound applications like machine learning, artificial intelligence, and so on, data movement from/to main memory and other chips (if any) contributes most to the application execution. Hence, a bandwidth/energy efficient interconnection design for such accelerators in the heterogeneous system is a necessity.

- Latency/Bandwidth Sensitivity: Similar to computation or memory intensive nature of applications, the latency or bandwidth sensitivity of an architecture imposes the primary constraints for off-chip (and also on-chip) interconnection design. The latency/bandwidth sensitivity of an architecture is determined by factors like predominantly serial/parallel execution at each core, spatial and temporal locality of memory accesses and instruction scheduling techniques, and so on. While most architectures fall into either category, significant challenges arise to meet conflicting requirements on shared off-chip interconnection, when architectures of different sensitivities are integrated in the heterogeneous system.

- Temporal Nature of Off-Chip Memory Accesses: The temporal behaviour of off-chip memory accesses, determined by the average number of memory accesses per compute, is dependent on application, computing architecture, reuse of cached data, and so on, and significantly impacts off-chip communication. Architectures with low volume, sporadically distributed off-chip memory accesses tolerate longer off-chip communication latencies and waiting time for resources through efficient instruction scheduling and data prefetching. However, dense temporal distribution or high volume of sparsely distributed memory accesses severely impact performance under long off-chip latency and interference from other inter-chip communication. Such architectures require prudent and efficient off-chip 
interconnection design to avoid high latency and energy consumption, interference for shared off-chip resources, and so on, to achieve optimal performance.

- Data sharing among the chips: Prevalence of data sharing among multiple chips require an inter-chip interconnection that can enable transmission of requests and updates to shared data across all the chips with low latency and low energy. Additionally, existence of communication with multiple chips require sharing of off-chip interconnection resources and leads to interference and increased communication latency. Hence, an interconnection that balances resource sharing and inter-chip communication performance is required.

- Real Time Constraints: In addition to achieving average performance, communication constraints in real-time applications are characterized by bounded and worst case performance to avoid any system failures. Traditional interconnection design focuses on optimizing average performance and has the best- and worst-case performances as secondary constraints. System design and consequently interconnection design for real-time architectures takes the opposite direction and further complicates inter-chip interconnection design when integrated with other architectures in the heterogeneous systems. Hence, additional constraints have to be imposed to meet worst case performance specifications, while optimizing average interconnect performance. These constraints can be in the form of minimizing sharing of off-chip interconnection resources, interference from other chips, channel access latency, and so on, in inter-chip wireless network take precedence to meet the real-time constraints.

In addition to these salient communication characteristics described above, application dependent behaviours like data reuse and memory subsystem specifications also impact the design of inter-chip wireless interconnection in heterogeneous multi-chip architectures. These heterogeneous inter-chip communication requirements can be met in a well-balanced and efficient manner through hybrid wireless interconnection design methodologies like multiple wireless channels, bandwidth division among different transceivers, multi-carrier modulations, and so on, along with combination of different radiation patters of wireless network.

\section{CONCLUSION}

A hybrid chip-to-chip wireless interconnection is proposed in this work to meet the demanding and diverse communication requirements of heterogeneous multi-chip architectures. The proposed wireless network combines omnidirectional and directional antennas operating at dual channels for efficient communication between any two chips. The omnidirectional wireless links in CPUs, operating at $f_{\text {omni }}$ frequency, provide low-latency, low-energy communication to any other system component. To provide sustained high-bandwidth communication for GPUs, its wireless network is implemented using directional wireless links between GPU and memory operating at $f_{\text {dir }}$ frequency. The proposed wireless interconnection speeds up application runtime by $1.15 \times$ to $24.42 \times$ for system with 4 CPUs and 4 GPUs as compared to baseline network with wired buses and PCIe switches. Using low-energy and low-energy wireless links, it saves packet energy by $94.4 \%$ and packet latency by $58.34 \%$ as compared to the baseline interconnection.

\section{REFERENCES}

[1] [n.d.]. AMD Accelerated Parallel Processing (APP) Software Development Kit (SDK). Retrieved from http://developer. amd.com/sdks/amdappsdk/.

[2] Levi Barnes. 2013. Multi-GPU programming. In Proceedings of the GPU Technology Conference. nVIDIA.

[3] Christian Bienia. 2011. Benchmarking Modern Multiprocessors. Ph.D. Dissertation. Princeton University.

[4] M. S. Birrittella, M. Debbage, R. Huggahalli, J. Kunz, T. Lovett, T. Rimmer, K. D. Underwood, and R. C. Zak. 2015. Intel ${ }^{\circledR}$ Omni-path architecture: Enabling scalable, high performance fabrics. In Proceedings of the 2015 IEEE $23 \mathrm{rd}$ Annual Symposium on High-Performance Interconnects. 1-9. DOI : https://doi.org/10.1109/HOTI.2015.22 
[5] M. S. Birrittella, M. Debbage, R. Huggahalli, J. Kunz, T. Lovett, T. Rimmer, K. D. Underwood, and R. C. Zak. 2016. Enabling scalable high-performance systems with the Intel Omni-path architecture. IEEE Micro 36, 4 (Jul. 2016), 3847. DOI : https://doi.org/10.1109/MM.2016.58

[6] S. Che, M. Boyer, J. Meng, D. Tarjan, J. W. Sheaffer, S. Lee, and K. Skadron. 2009. Rodinia: A benchmark suite for heterogeneous computing. In Proceedings of the 2009 IEEE International Symposium on Workload Characterization (IISWC'09). 44-54. DOI : https://doi.org/10.1109/IISWC.2009.5306797

[7] Ian Cutress. 2017. AMD's Future in Servers: New 7000-series CPUs Launched and EPYC Analysis. Retrieved from https://www.anandtech.com/show/11551/amds-future-in-servers-new-7000-series-cpus-launched-and-epycanalysis/2.

[8] Manish Deo. 2017. Enabling Next-Generation Platforms Using Intel's 3D System-in-Package Techology. Technical Report. Intel.

[9] S. H. Gade and S. Deb. 2017. HyWin: Hybrid wireless NoC with sandboxed sub-networks for CPU/GPU architectures. IEEE Trans. Comput. 66, 7 (Jul. 2017), 1145-1158. DOI : https://doi.org/10.1109/TC.2016.2643668

[10] S. H. Gade, S. Garg, and S. Deb. 2017. OFDM based high data rate, fading resilient transceiver for wireless networkson-chip. In Proceedings of the 2017 IEEE Computer Society Annual Symposium on VLSI (ISVLSI'17). 483-488. DOI : https://doi.org/10.1109/ISVLSI.2017.90

[11] Sri Harsha Gade, Shobha Sundar Ram, and Sujay Deb. 2019. Millimeter wave wireless interconnects in deep submicron chips: Challenges and opportunities. Integration 64 (2019), 127-136. DOI : https://doi.org/10.1016/j.vlsi.2018.09.004

[12] S. H. Gade, S. S. Rout, M. Sinha, H. K. Mondal, W. Singh, and S. Deb. 2018. A utilization aware robust channel access mechanism for wireless NoCs. In Proceedings of the 2018 IEEE International Symposium on Circuits and Systems (ISCAS'18). 1-5. DOI : https://doi.org/10.1109/ISCAS.2018.8351698

[13] S. H. Gade, M. Sinha, S. S. Rout, and S. Deb. 2018. Enabling reliable high throughput on-chip wireless communication for many core architectures. In Proceedings of the 2018 IEEE Computer Society Annual Symposium on VLSI (ISVLSI'18). 591-596. DOI : https://doi.org/10.1109/ISVLSI.2018.00113

[14] Mark Harris. 2013. Unified Memory in CUDA 6. Retrieved from https://devblogs.nvidia.com/unified-memoryin-cuda-6/.

[15] Intel. 2016. Intel omni-path 4.8 Tbps switch ASIC and platform. In Proceedings of the 2016 IEEE Hot Chips 28 Symposium (HCS'16). 1-17. DOI : https://doi.org/10.1109/HOTCHIPS.2016.7936213

[16] A. Karkar, T. Mak, K. F. Tong, and A. Yakovlev. 2016. A survey of emerging interconnects for on-chip efficient multicast and broadcast in many-cores. IEEE Circ. Syst. Mag. 16, 1 (Firstquarter 2016), 58-72. DOI : https://doi.org/10.1109/ MCAS.2015.2510199

[17] G. Kim, M. Lee, J. Jeong, and J. Kim. 2014. Multi-GPU system design with memory networks. In Proceedings of the 2014 47th Annual IEEE/ACM International Symposium on Microarchitecture. 484-495. DOI : https://doi.org/10.1109/MICRO. 2014.55

[18] Akhilesh Kumar and Malay Trivedi. 2017. Intel Xeon Scalable Processor Architecture Deep Dive. Retrieved from http://www.primeline-solutions.de/files/intel-xeon-scalable-architecture-deep-dive1.pdf.

[19] Byung-Jae Kwak, Nah-Oak Song, and L. E. Miller. 2005. Performance analysis of exponential backoff. IEEE/ACM Trans. Netw. 13, 2 (Apr. 2005), 343-355. DOI : https://doi.org/10.1109/TNET.2005.845533

[20] S. Laha, S. Kaya, D. W. Matolak, W. Rayess, D. DiTomaso, and A. Kodi. 2015. A new frontier in ultralow power wireless links: Network-on-chip and chip-to-chip interconnects. IEEE Trans. Comput.-Aid. Des. Integr. Circ. Syst. 34, 2 (Feb. 2015), 186-198. DOI : https://doi.org/10.1109/TCAD.2014.2379640

[21] J. H. Lau. 2011. Evolution, challenge, and outlook of TSV, 3D IC integration and 3d silicon integration. In Proceedings of the 2011 International Symposium on Advanced Packaging Materials (APM'11). 462-488. DOI : https://doi.org/10.1109/ ISAPM.2011.6105753

[22] Jason Lawley. 2014. Understanding Performance of PCI Express Systems. Whitepaper. Xilinx.

[23] L. Li, P. Ton, M. Nagar, and P. Chia. 2017. Reliability challenges in 2.5D and 3D IC integration. In Proceedings of the 2017 IEEE 67th Electronic Components and Technology Conference (ECTC'17). 1504-1509. DOI : https://doi.org/10.1109/ ECTC.2017.208

[24] Gabriel H. Loh. 2008. 3D-stacked memory architectures for multi-core processors. In Proceedings of the 35th Annual International Symposium on Computer Architecture (ISCA'08). IEEE Computer Society, Los Alamitos, CA, 453-464. DOI : https://doi.org/10.1109/ISCA.2008.15

[25] R. Mahajan, R. Sankman, N. Patel, D. Kim, K. Aygun, Z. Qian, Y. Mekonnen, I. Salama, S. Sharan, D. Iyengar, and D. Mallik. 2016. Embedded multi-die interconnect bridge (EMIB)-A high density, high bandwidth packaging interconnect. In Proceedings of the 2016 IEEE 66th Electronic Components and Technology Conference (ECTC'16). 557-565. DOI : https://doi.org/10.1109/ECTC.2016.201

[26] H. Matsutani, M. Koibuchi, I. Fujiwara, T. Kagami, Y. Take, T. Kuroda, P. Bogdan, R. Marculescu, and H. Amano. 2014. Low-latency wireless 3D NoCs via randomized shortcut chips. In Proceedings of the 2014 Design, Automation Test in Europe Conference Exhibition (DATE'14). 1-6. DOI : https://doi.org/10.7873/DATE.2014.286 
[27] H. K. Mondal, S. H. Gade, S. Kaushik, and S. Deb. 2017. Adaptive multi-voltage scaling with utilization prediction for energy-efficient wireless NoC. IEEE Trans. Sust. Comput. 2, 4 (Oct. 2017), 382-395. DOI : https://doi.org/10.1109/ TSUSC.2017.2742219

[28] Hemanta Kumar Mondal, Sri Harsha Gade, Raghav Kishore, and Sujay Deb. 2017. P2NoC: Power- and performanceaware NoC architectures for sustainable computing. Sust. Comput. Inf. Syst. 16 (2017), 25-37. DOI : https://doi.org/10. 1016/j.suscom.2017.08.005

[29] J. Nickolls and W. J. Dally. 2010. The GPU computing era. IEEE Micro 30, 2 (Mar. 2010), 56-69. DOI : https://doi.org/10. 1109/MM.2010.41

[30] nVIDIA. 2017. NVIDIA DGX-1 System Architecture-The Fastest Platform for Deep Learning. nVIDIA. Retrieved from http://www.nvidia.com/dgx1.

[31] nVIDIA. 2018. NVIDIA NVSWITCH-The World's Highest Bandwidth On-Node Switch. Retrieved from http://images. nvidia.com/content/pdf/nvswitch-technical-overview.pdf.

[32] A. Samaiyar, S. S. Ram, and S. Deb. 2014. Millimeter-wave planar log periodic antenna for on-chip wireless interconnects. In Proceedings of the 8th European Conference on Antennas and Propagation (EuCAP'14). 1007-1009. DOI : https://doi.org/10.1109/EuCAP.2014.6901936

[33] M. S. Shamim, M. M. Ahmed, N. Mansoor, and A. Ganguly. 2017. Energy-efficient wireless interconnection framework for multichip systems with in-package memory stacks. In Proceedings of the 2017 30th IEEE International System-onChip Conference (SOCC'17). 357-362. DOI : https://doi.org/10.1109/SOCC.2017.8226077

[34] M. S. Shamim, N. Mansoor, R. S. Narde, V. Kothandapani, A. Ganguly, and J. Venkataraman. 2017. A wireless interconnection framework for seamless inter and intra-chip communication in multichip systems. IEEE Trans. Comput. 66, 3 (Mar. 2017), 389-402. DOI : https://doi.org/10.1109/TC.2016.2605093

[35] R. Ubal, B. Jang, P. Mistry, D. Schaa, and D. Kaeli. 2012. Multi2Sim: A simulation framework for CPU-GPU computing. In Proceedings of the 2012 21st International Conference on Parallel Architectures and Compilation Techniques (PACT'12). 335-344.

[36] Verizon. 2017. State of the Market: Internet of Things 2017. Retrieved from https://www.verizon.com/about/sites/ default/files/Verizon-2017-State-of-the-Market-IoT-Report.pdf.

[37] WikiChip. 2018. Infinity Fabric (IF)-AMD. Retrieved from https://en.wikichip.org/wiki/amd/infinity_fabric.

[38] S. C. Woo, M. Ohara, E. Torrie, J. P. Singh, and A. Gupta. 1995. The SPLASH-2 programs: Characterization and methodological considerations. In Proceedings of the 22nd Annual International Symposium on Computer Architecture. 24-36. DOI : https://doi.org/10.1109/ISCA.1995.524546

[39] X. Wu, Y. Ye, J. Xu, W. Zhang, W. Liu, M. Nikdast, and X. Wang. 2014. UNION: A unified inter/intrachip optical network for chip multiprocessors. IEEE Trans. VLSI Syst. 22, 5 (May 2014), 1082-1095. DOI : https://doi.org/10.1109/ TVLSI.2013.2263397

[40] X. Yu, J. Baylon, P. Wettin, D. Heo, P. P. Pande, and S. Mirabbasi. 2014. Architecture and design of multichannel millimeter-wave wireless NoC. IEEE Des. Test 31, 6 (Dec. 2014), 19-28. DOI : https://doi.org/10.1109/MDAT.2014 2322995

[41] Xiaowu Zhang, Jong Kai Lin, Sunil Wickramanayaka, Songbai Zhang, Roshan Weerasekera, Rahul Dutta, Ka Fai Chang, King-Jien Chui, Hong Yu Li, David Soon Wee Ho, Liang Ding, Guruprasad Katti, Suryanarayana Bhattacharya, and Dim-Lee Kwong. 2015. Heterogeneous 2.5D integration on through silicon interposer. Appl. Phys. Rev. 2, 2 (2015), 021308. DOI : https://doi.org/10.1063/1.4921463 arXiv:https://doi.org/10.1063/1.4921463

Received December 2018; revised April 2019; accepted June 2019 